\title{
SRSF10-mediated IL1RAP alternative splicing regulates cervical cancer oncogenesis via mIL1RAP-NF-KB-CD47 axis
}

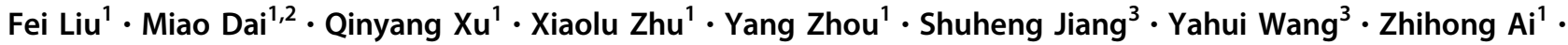 \\ Li Ma ${ }^{1}$ Y Yanli Zhang ${ }^{3} \cdot$ Lipeng $\mathrm{Hu}^{3} \cdot$ Qin $\mathrm{Yang}^{3} \cdot$ Jun $\mathrm{Li}^{3} \cdot$ Shujie Zhao ${ }^{4}$. Zhigang Zhang ${ }^{3} \cdot$ Yincheng Teng ${ }^{1}$
}

Received: 26 September 2017 / Revised: 6 December 2017 / Accepted: 14 December 2017 / Published online: 12 February 2018

(c) The Author(s) 2018. This article is published with open access

\begin{abstract}
High-risk human papillomavirus oncoproteins E6 and E7 are the major etiological factors of cervical cancer but are insufficient for malignant transformation of cervical cancer. Dysregulated alternative splicing, mainly ascribed to aberrant splicing factor levels and activities, contributes to most cancer hallmarks. However, do E6 and E7 regulate the expression of splicing factors? Does alternative splicing acts as an "accomplice" of E6E7 to promote cervical cancer progression? Here, we identified that the splicing factor SRSF10, which promotes tumorigenesis of cervix, was upregulated by E6E7 via E2F1 transcriptional activation. SRSF10 modulates the alternate terminator of interleukin-1 receptor accessory protein exon 13 to increase production of the membrane form of interleukin-1 receptor accessory protein. SRSF10-mediated mIL1RAP upregulates the expression of the "don't eat me" signal CD47 to inhibit macrophage phagocytosis by promoting nuclear factor- $\mathrm{kB}$ activation, which is pivotal in inflammatory, immune, and tumorigenesis processes. Altogether, these data reveal a close relationship among HPV infection, alternative splicing and tumor immune evasion, and also suggests that the SRSF10mIL1RAP-CD47 axis could be an attractive therapeutic target for the treatment of cervical cancer.
\end{abstract}

Fei Liu and Miao Dai contributed equally to this work.

Electronic supplementary material The online version of this article (https://doi.org/10.1038/s41388-017-0119-6) contains supplementary material, which is available to authorized users.

\section{Zhigang Zhang \\ zzhang@shsci.org \\ Yincheng Teng \\ ycteng@sjtu.edu.cn}

1 Department of Gynecology and Obstetrics, Shanghai Jiao Tong University Affiliated Sixth People's Hospital, Shanghai Jiao Tong University School of medicine, Shanghai, China

2 Department of Gynecologic Oncology, Hunan Cancer Hospital, The Affiliated Cancer Hospital of Xiangya School of Medicine, Central South University, Changsha, China

3 State Key Laboratory for Oncogenes and Related Genes, Shanghai Cancer Institute, Shanghai Jiao Tong University, Shanghai, China

4 Department of Orthopedics, The First Affiliated Hospital of Nanjing Medical University, Nanjing, China

\section{Introduction}

Alternative splicing (AS) drives proteome diversity and affords a significant evolutionary advantage by generating multiple different mRNAs and downstream proteins from a single gene through the inclusion or exclusion of specific exons [1, 2]. AS is often regulated at the tissue level, whereas it can be aberrantly regulated by cancer cells to their advantage [3]. The splicing pattern of numerous genes is altered as cells move through the oncogenic process of gaining proliferative, antiapoptotic, invasive, metastatic, and angiogenic properties, becoming free from growth factor dependence and growth suppression, altering their metabolism and acquiring mechanisms of immune escape $[4,5]$. AS elicits control over the major hallmarks of cancer toward more aggressive invasive cancer phenotypes. This process is generally regulated by splicing factors, which are dysregulated in cancer and might contribute to positive feedback loops that drive cancer progression $[6,7]$. Considering that a relatively small number of splicing factors drive multiple oncogenic processes, understanding how splicing factors are regulated could lead to the development of a new class of anticancer therapeutics [8]. 
a

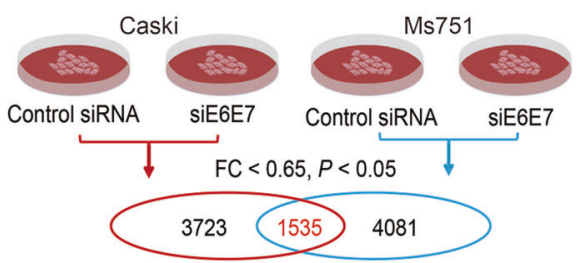

b

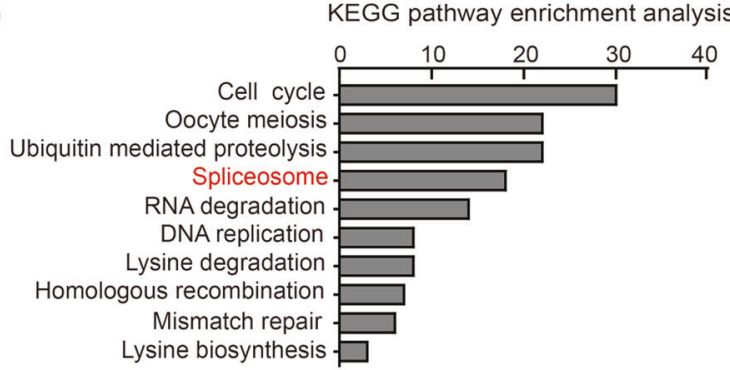

e

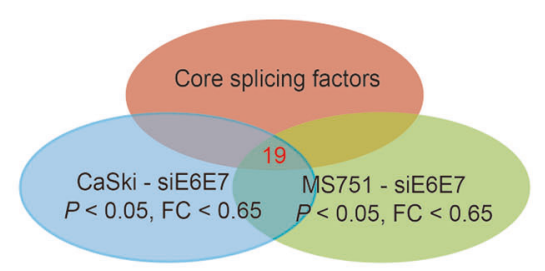

C

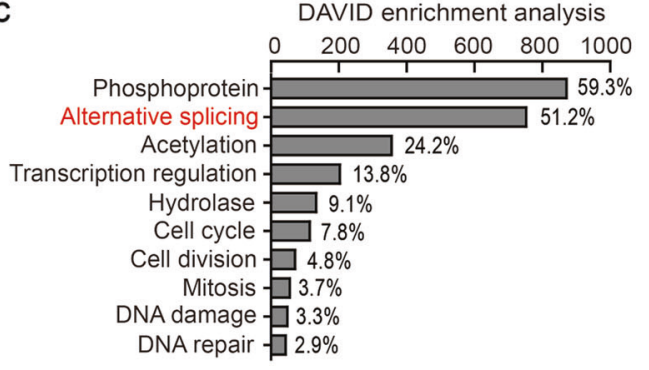

d

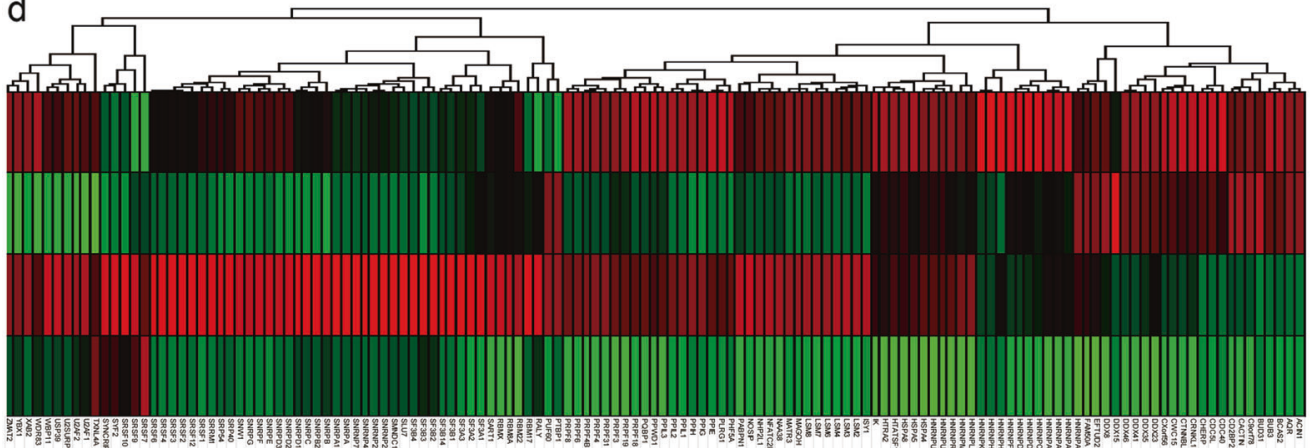

CaSki - Control siRNA

CaSki - siE6E7

MS751 - Control siRNA

MS751 - siE6E7

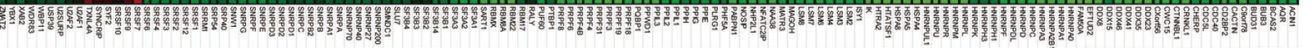

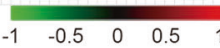

$f$

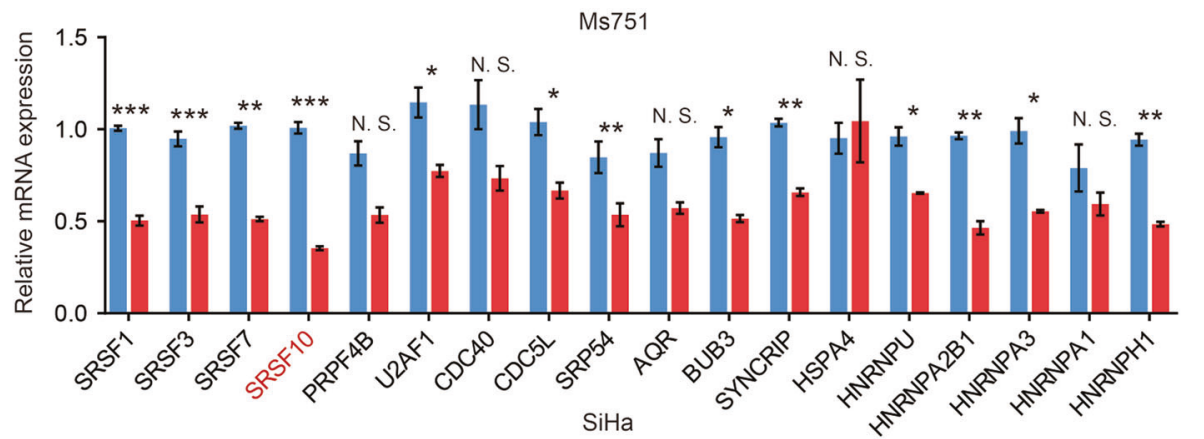

Control siRNA
siE6E7

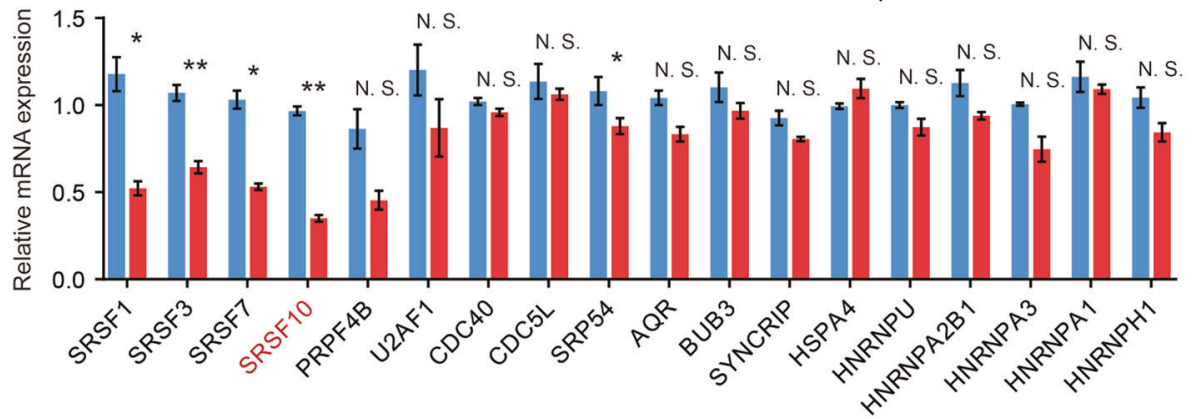

Cervical cancer (CC) is one of the leading causes of cancer death among women, especially in developing countries [9, 10]. Repeated and persistent high-risk HPV infection are considered the major etiologic contributor, with HPV DNA identified in 95\% of malignant cervical lesions [11]. Multiple molecular studies indicate that HPV- 
Fig. 1 E6 and E7 regulate the expression of splicing factors in CC cells. a Summary of microarray analyses. In all, microarray analyses were performed in Caski and Ms751 cells transiently transfected with either siE6E7 or control siRNA to screen the differentially expressed genes. A total of 1535 genes were identified after overlap in Caski and Ms751 cells. b Top significant pathways for overlapped differentially expressed genes in E6E7-knockdown cells analyzed by KEGG pathway enrichment analysis. Spliceosome ranked fourth. c Top significant pathways for overlapped differentially expressed genes in E6E7knockdown cells analyzed by DAVID enrichment analysis. Alternative splicing is the second pathway affected by E6E7 knockdown. d The heatmap of differentially expressed genes in AS pathway in Caski and Ms751 groups. e The schematic diagram of differentially expressed core splicing factors. In all, 19 genes were identified by overlapping differentially expressed genes related to AS pathway in microarray and core splicing factors in Ref. [16]. f Validation of the microarray results by RT-PCR of the 19 core splicing factors in Ms751 and $\mathrm{SiHa}$ cells. The expression of HNRPDL is undetectable. The experiments were repeated three times, and the results are shown as mean \pm s.d. $* P<0.05 ; * * P<0.01 ; * * * P<0.001$ (Student's $t$-test)

mediated $\mathrm{CC}$ is mainly owing to the oncogenic activities of the viral early proteins E6 and E7 [12]. E6 induces the degradation of p53 via the ubiquitin pathway [13], whereas E7 associates with the retinoblastoma family of proteins ( $\mathrm{pRb}, \mathrm{p} 107$, and $\mathrm{p} 130)$ and disrupts their association with the E2F family of transcription factors [14]. P53 and E2Fs in turn activate the transcription of a group of genes and thus contribute to tumorigenesis.

Based on the above theory, splicing factors act as floodgates in oncogenic processes, and E6E7 are prerequisites for CC. However, do E6 and E7 regulate the expression of splicing factors? Does AS act as a "co-conspirator" of E6E7 in CC development? In this study, we aimed to investigate the relationship among E6E7, aberrant $\mathrm{AS}$ and CC. We found that E6E7 regulated the expression of splicing factors in CC, especially SRSF10. SRSF10 was increased via transcriptional activation of the E6E7-E2F1 axis and promoted tumorigenesis in cervix. MIL1RAP, a membrane isoform of IL1RAP, was splicing-regulated by SRSF10 and involved in the oncogenic effect of SRSF10. SRSF10-mIL1RAP upregulated the expression of CD47 to inhibit macrophage phagocytosis by promoting IL1- $\beta$ induced nuclear factor- $\kappa \mathrm{B}(\mathrm{NF}-\kappa \mathrm{B})$ activation. These findings provide new insights into the connections among HPV infection, aberrant AS and oncogenesis in cervix.

\section{Results}

\section{HR-HPV viral oncoproteins E6 and E7 regulate the expression of splicing factors}

We first investigated whether E6E7 could regulate the expression of splicing factors in CC. To achieve this goal, we used siRNAs targeted against E6E7 and performed a microarray analysis using total RNA prepared from siE6E7 and control cells to identify differentially expressed genes (Fig. 1a) [15]. The Kyoto Encyclopedia of Genes and Genomes (KEGG) pathway and the Database for Annotation, Visualization and Integrated Discovery (DAVID) enrichment analyses revealed that these genes were significantly associated with spliceosome or AS processes, which suggested that E6E7 could regulate the expression of splicing factors in CC cells (Fig. 1b-d and Supplementary Table S1). Furthermore, real-time-polymerase chain reaction (RT-PCR) was performed to validate the differentially expressed core splicing factors in both E6E7 knockdown cell lines (Fig. 1e, f) [16]. As shown in Fig. 1f, the most significantly downregulated gene was SRSF10 with more than a twofold decrease after E6E7 knockdown. Altogether, these results suggest that the viral oncoproteins E6E7 can regulate the expression of splicing factors in $\mathrm{CC}$, especially SRSF10.

\section{Upregulation of SRSF10 is implicated in the carcinogenesis of human HPV 16/18-positive CC}

As SRSF10 is regulated by E6E7, we next assessed the role of SRSF10 in the carcinogenesis of CC. To prove this point, we first analyzed the mRNA levels of SRSF10 in human $\mathrm{CC}$ tissues using published data sets from Oncomine and the Gene Expression Omnibus database (GEO) [17-20]. In silico analysis of four independent data sets demonstrated that SRSF10 was upregulated in CC tissues compared with normal tissues (Fig. 2a and Supplementary Fig. S1a-c). Interestingly, SRSF10 expression was much higher in HPV16/18-positive CCs than in HPV-negative ones (Fig. $2 \mathrm{~b}$ and Supplementary Fig. S1d). More importantly, the expression levels of SRSF10 gradually increased with the malignant transformation from normal cervical epithelia to cervical intraepithelial neoplasias (CINs) to CCs (Fig. 2c). For more evidence, we next examined protein levels of SRSF10 in a CC tissue array by IHC. Consistent with the mRNA level, the protein level of SRSF10 also increased from normal cervical tissues to CINs and further to CCs in a sequential manner. Together, these observations suggest that upregulation of SRSF10 might have a role in the progression of HPV16/18-positive CC.

We further investigated the role of SRSF10 in CC in a xenograft mouse model. Ms751 and $\mathrm{SiHa}$ cells stably expressing SRSF10 shRNA were injected into the flanks of 4-week-old nude mice. Knockdown of SRSF10 significantly decreased the tumor growth rate and tumor weight (Fig. 2f-h and Supplementary Fig. S2a-c). Overall, these data demonstrate the cancer-promoting function of SRSF10 in HPV16/18-positive CC. 

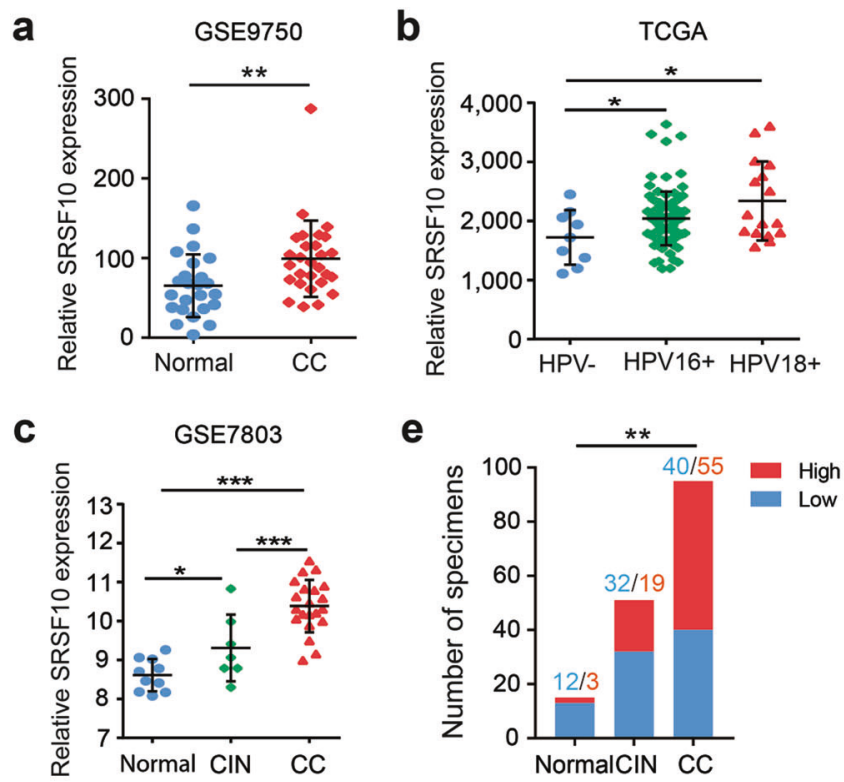

f

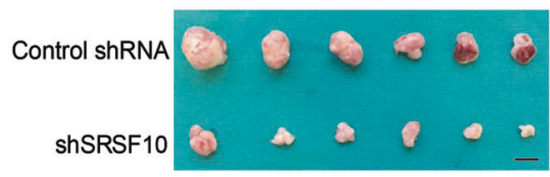

Fig. 2 The upregulated expression of SRSF10 is associated with progression of CC. a Analyses of SRSF10 mRNA levels in normal cervical tissues $(n=24)$ and CC $(n=33)$ in GSE9750 data set. b Point diagrams comparing SRSF10 mRNA levels in HPV-negative CCs $(n$ =9), HPV 16-positive CCs $(n=94)$ and HPV 18-positive CCs $(n=$ 17) in TCGA. c Analyses of SRSF10 mRNA levels in normal cervical tissues $(n=10)$, CINs $(n=7)$ and CC $(n=21)$ in GSE7803 data set. d Representative photographs of the SRSF10 immunoreactivity in normal tissue, CIN and CCs in TMA (scale bar: $200 \mu \mathrm{m}$ ). e The constituent ratio of SRSF10 expression assessed by blinded IHC analyses in normal tissues $(n=15)$, CINs $(n=51)$ and CCs $(n=95)$. f

\section{The expression of SRSF10 is regulated by the transcription factor E2F1 in HPV16/18-positive CC}

The mechanism by which E6E7 upregulates the expression of SRSF10 is currently unknown. The main induction of HPV-related CC are E6 resulting in proteolysis of p53 and $\mathrm{E} 7$ inactivating $\mathrm{Rb}$ followed by an increase in E2Fs [13, 14]. Therefore, we reasonably hypothesized that E6E7 might regulate the expression of SRSF10 through the transcription factors p53 or E2Fs. We first used the websites JASPAR (http://jaspar.genereg.net/) and PROMO (http://a lggen.lsi.upc.es/cgi-bin/promo_v3/promo/promoinit.cgi? $\operatorname{dirDB}=\mathrm{TF} \_8.3$ ) for a primary prediction. Both websites showed E2F1-binding elements in the SRSF10 promoter. However, there were no uniform p53 binding elements in the SRSF10 promoter. Thus, we focused on E2F1 for further verification. We designed 10 primer sets covering the individual SRSF10 binding motifs for PCR after chromatin d

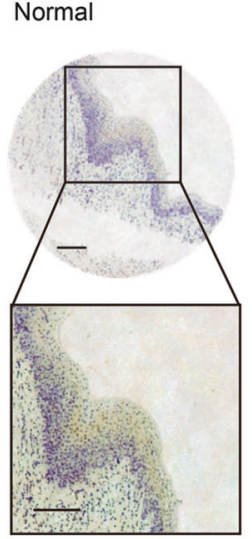

CIN

Cervical CA

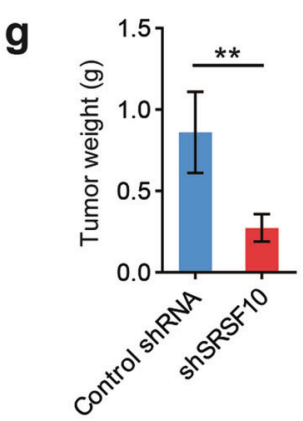

h

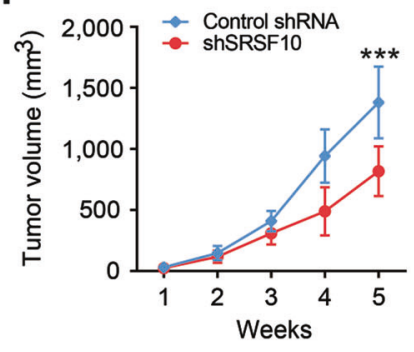

Ms751 cells were infected with either retrovirus expressing SRSF10knockdown shRNA (shSRSF10) or control vector (Control shRNA) and selected for puromycin resistance. Stable Ms751 cells were injected into nude mice, which were randomly divided into two groups $(n=6)$. Mice were killed at 5 weeks after injection. Tumors were excised from the mice and weighed (scale bar: $1 \mathrm{~cm}$ ). $\mathbf{g}$ Tumor weight. Results are shown as mean \pm s.d. of the tumor weight. $\mathbf{h}$ Time-course of xenograft growth. The tumor volumes of mice described in $\mathbf{f}$ were measured every week. Each point represents the mean \pm s.d. of the tumors. $* P<0.05 ; * * P<0.01 ; * * * P<0.001$ (Student's $t$-test)

immunoprecipitation (ChIP) to confirm the binding of E2F1 to the SRSF10 promoter (Fig. 3a). As shown in Fig. 3b, E2F1-binding was detected at the SRSF10 promoter sites 5 and 9 in both Ms751 and SiHa cells. To provide additional evidence, we cloned the intact SRSF10 promoter into luciferase constructs. When introduced into Ms751 and $\mathrm{SiHa}$ cells, the constructs with an intact SRSF10 promoter showed increased luciferase activity, suggesting a direct transcriptional modulation of E2F1 over the SRSF10 promoter (Fig. 3d, e). In addition, reporter assays revealed that mutations of predicted binding site 5 or 9 abrogated responsiveness to E2F1 in Ms751 and SiHa cells, demonstrating the specificity of $-362,-543$, and -1688 -bp regions as the E2F1-binding site in the SRSF10 promoter (Fig. 3c-e).

Next, we examined the regulatory capacity of E2F1 on SRSF10 expression by siRNA targeted against E2F1. As shown in Fig. 3f, E2F1 knockdown significantly 

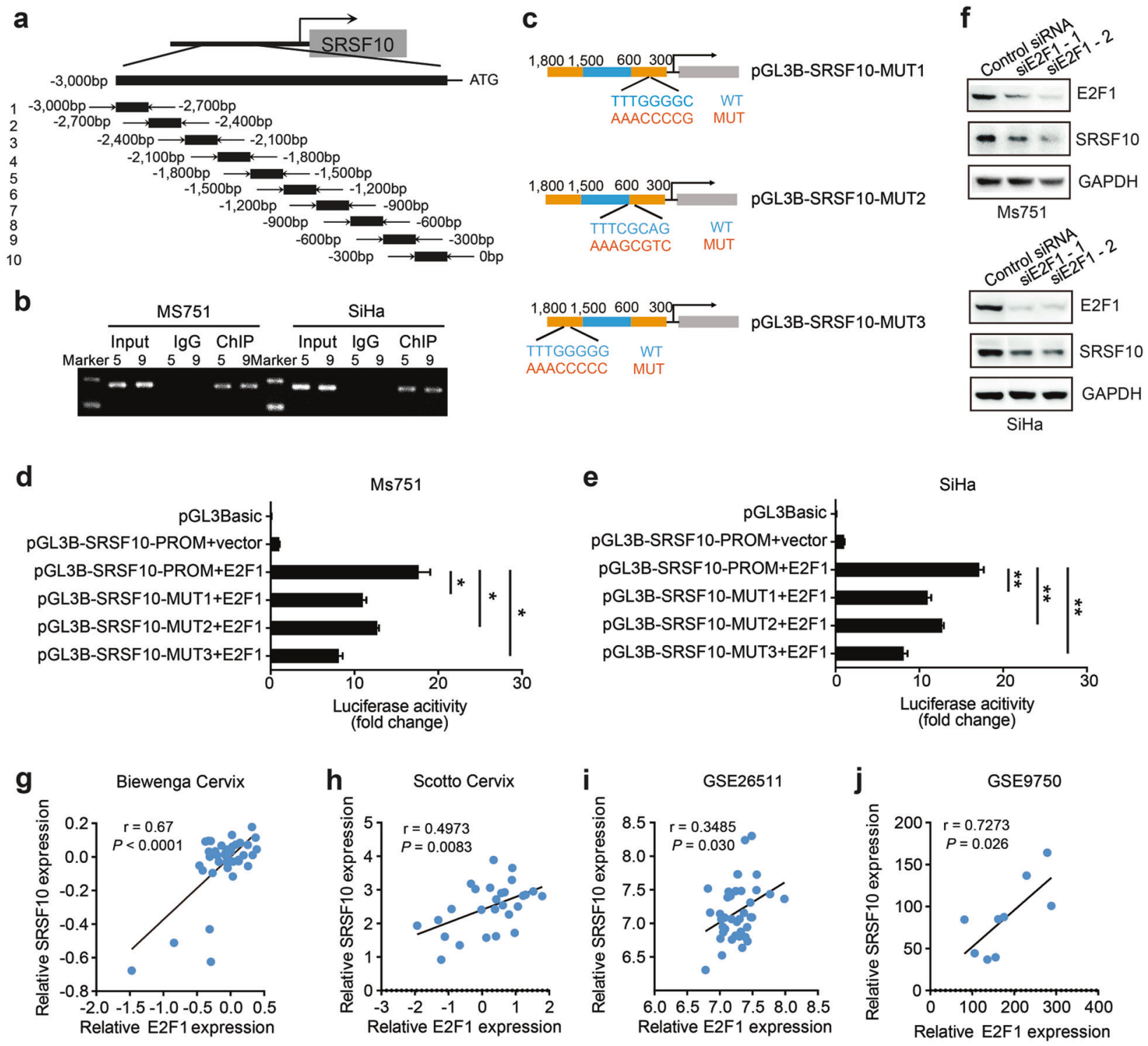

Fig. 3 SRSF10 is the direct target of E2F1 in HPV16/18-positive CC. a Sketch map of primers for SRSF10 promoter sequences. Ten primer sets with a 300-bp partition were designed for PCR to test the direct binding of E2F1 to the SRSF10 promoter and the primer pairs produced 10 fragments of $300 \mathrm{bp}$. b The chromatin DNA of Ms751 and $\mathrm{SiHa}$ cells was chromatinimmunoprecipitated (ChIP) with HA antibody. Sonicated input DNA and IgG were used as control. Amplification of the SRSF10 promoter sequence from ChIP DNA validated the binding of E2F1 to SRSF10 promoter site 5 and 9. c Diagram of SRSF10 mutant plasmids. d, e Verification of SRSF10 as an E2F1 target via luciferase reporter assays. Constructs with an intact SRSF10 promoter resulted in enhanced luciferase activities in E2F1-expressing

Ms751 and SiHa cells, whereas those carrying either mutant site 5 or 9 resulted in strongly repressed luciferase activities. $\mathbf{f}$ Western blots for E2F1 and SRSF10 in Ms751 and SiHa cells transiently transfected with either E2F1-knockdown siRNAs (siE2F1-1, siE2F1-2) or control siRNA. GAPDH was used as a loading control. E2F1 knockdown decreased the expression of SRSF10 in both cells. $\mathbf{g - j}$-j There was a positive correlation between the expression levels of E2F1 and SRSF10 in four independent CC data sets as described in Fig. 2 and Supplementary Fig. S2. The ordinate of Figs. $3 \mathrm{~g}$, h represented the $\log 2$ median-centered intensity. The relationships between these two variables were determined by Pearson's correlation coefficients. $* P<$ $0.05 ; * * P<0.01 ; * * * P<0.001$

downregulated the expression of SRSF10 in both CC cells. Further analysis of the clinical human CC data sets revealed a consistent positive correlation between E2F1 and SRSF10 expression (Fig. 3g-j). Collectively, these results indicate that E6E7 upregulates the expression of SRSF10 via E2F1 transcriptional activation in HPV16/18positive CC.

\section{SRSF10 promotes the production of mIL1RAP via regulating the AS of IL1RAP exon 13}

Considering that SRSF10 acts as a critical splicing regulator, we logically asked whether SRSF10 executed its oncogenetic effects by regulating AS in CC. To test this hypothesis, we first analyzed SRSF10-related AS events in 
a

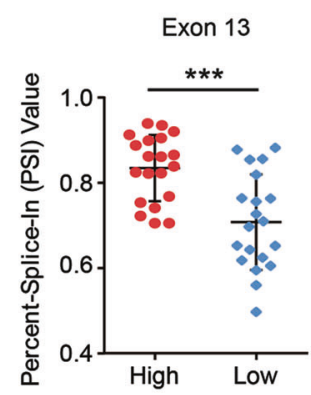

b

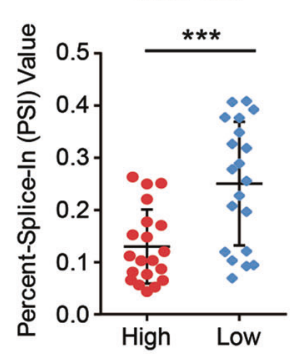

k

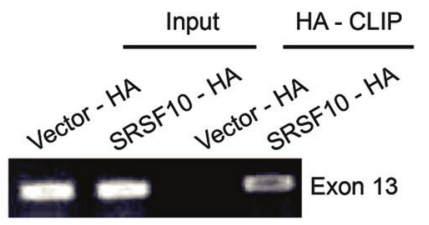

C

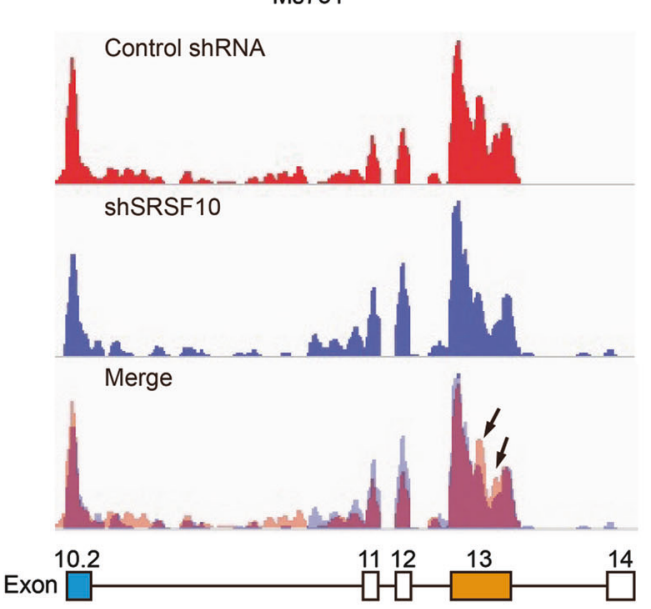

e

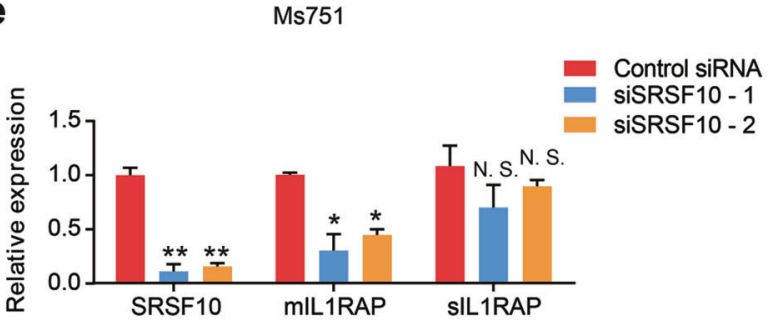

g
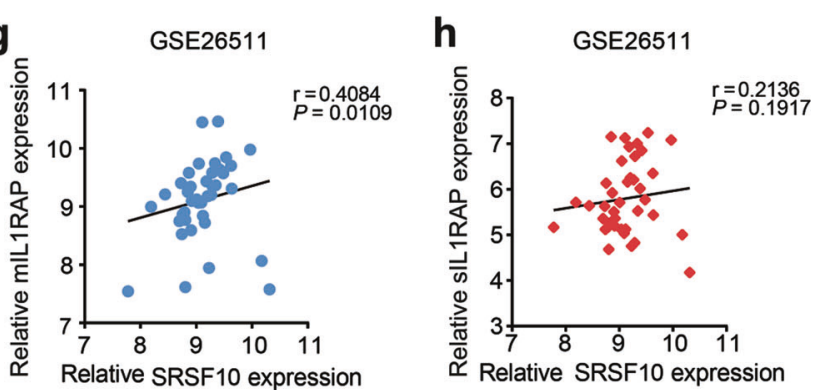

Fig. 4 SRSF10 promotes the generation of mIL1RAP in CC. $(\mathbf{a}, \mathbf{b}$ Quantification of AT of IL1RAP exon 13 and 10.2 in the high SRSF10 group $(n=20)$ and low SRSF10 group $(n=20)$ in the TCGA CESC data set (described in Supplementary Fig. S2A) measured as the PSI (percent splicing index). c, d Schematic diagram of RNA-seq reads covering SRSF10 exons 10.2-14 in shSRSF10 and control shRNA CC cells. In the merged diagram, reads of control shRNA are showed as orange, shSRSF10 as blue and overlap as purple. The arrow showed the exclusion of exon 13 after SRSF10 knockdown. e, f The AS of mIL1RAP and SIL1RAP affected by SRSF10 knockdown were examined in Ms751 and SiHa cells. Ms751 and SiHa cells were d
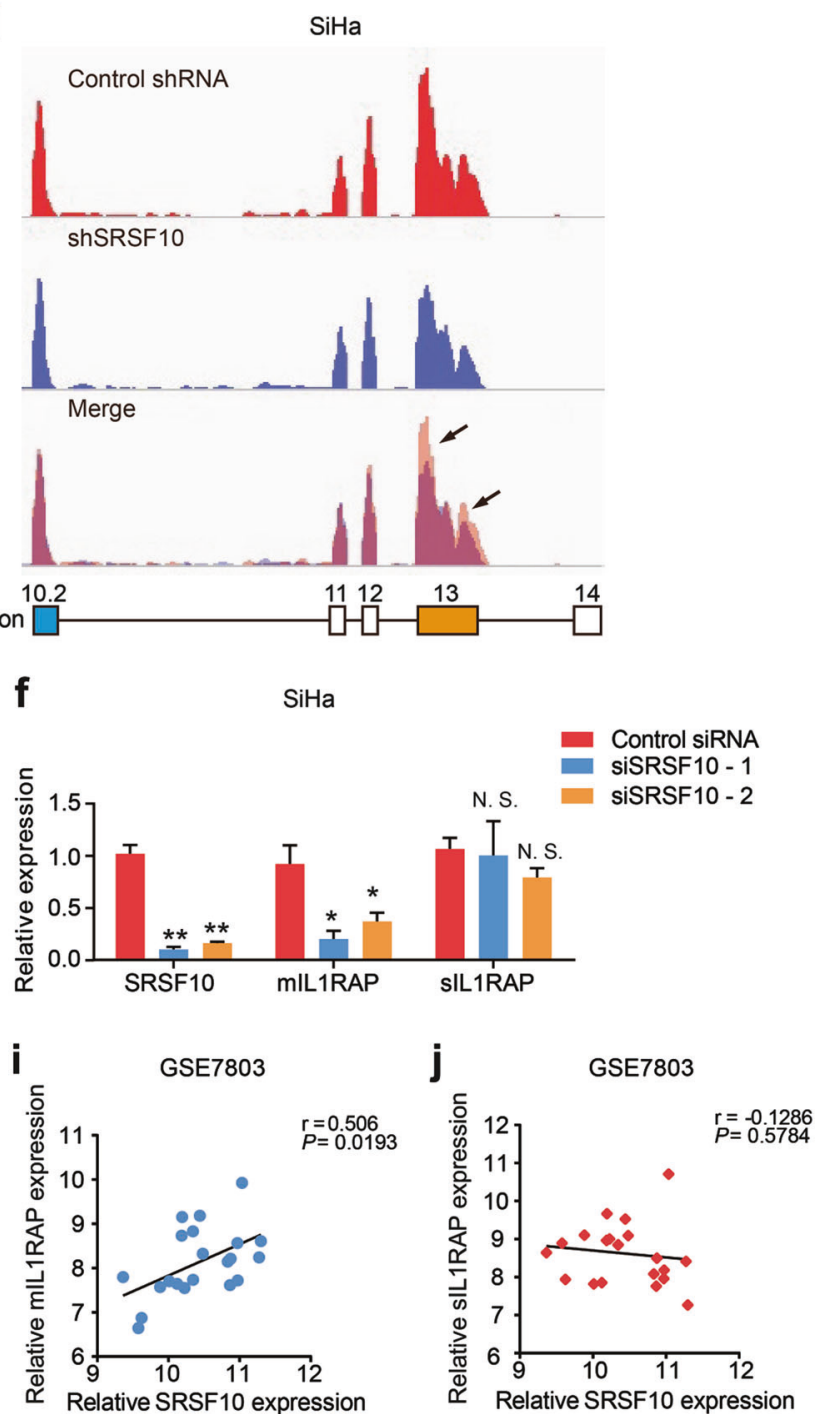

transiently transfected with either SRSF10-knockdown siRNAs (siSRSF10-1, siSRSF10-2) or control siRNA. RNAs extracted from siSRSF10 and control siRNA cells were examined by RT-PCR. g-j The correlation analysis between SRSF10 and mIL1RAP or sIL1RAP was observed in CC samples of GSE26511 $(n=28)$ and GSE7803 $(n$ $=21$ ). $\mathbf{k}$ In vitro CLIP from SiHa cells with primer pairs complementary to human IL1RAP exon 13 by RT-PCR. Siha cells were transiently transfected with either HA-tagged SRSF10 (SRSF10-HA) or empty-vector control (Vector-HA). $* P<0.05$; $* * P<0.01$; $* * * P<$ 0.001 
a

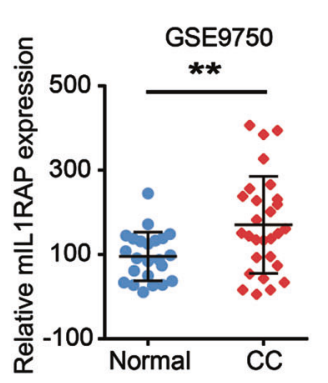

b

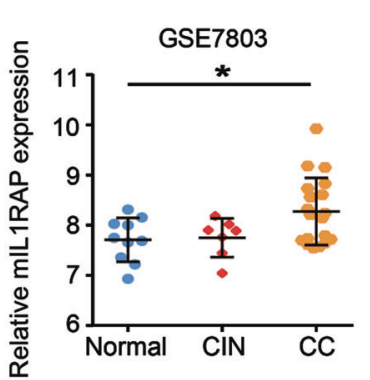

C

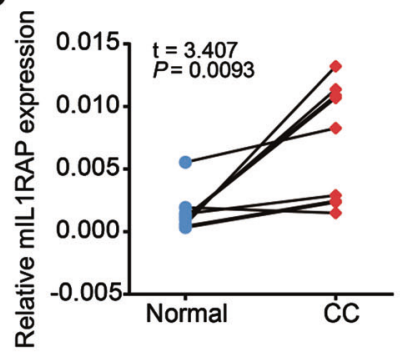

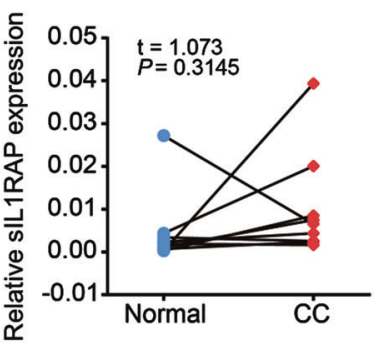

d

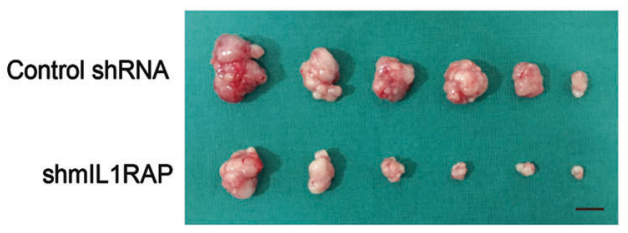

g

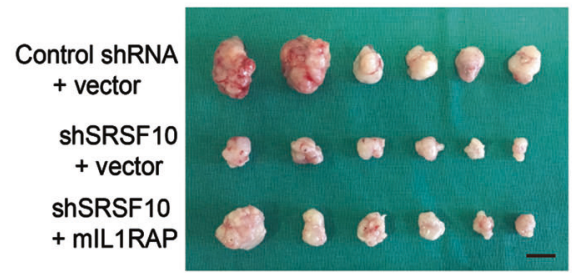

e

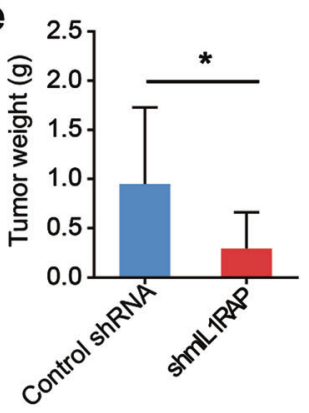

h

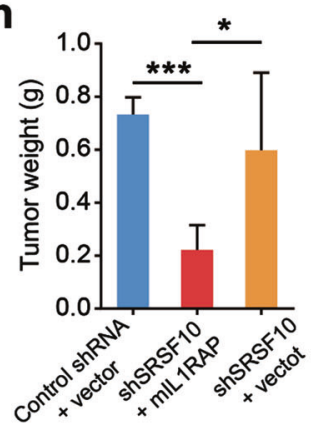

f

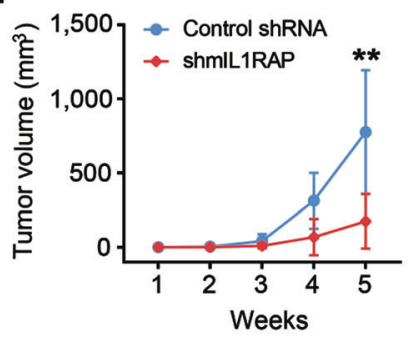

i

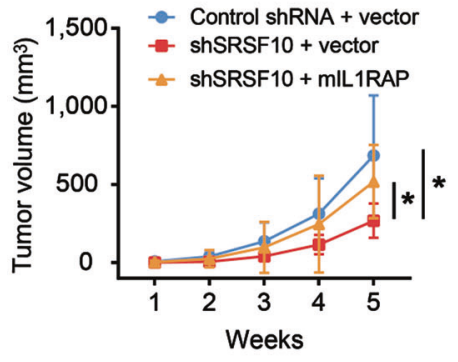

Fig. 5 MIL1RAP participates in SRSF10-mediated CC tumorigenesis. a Analysis of mIL1RAP mRNA levels in normal cervical tissues and CCs in GSE9750 indicated in Fig. 2a. b Analysis of mIL1RAP mRNA levels in normal cervical tissues, CINs, and CCs in GSE7803 indicated in Fig. 2c. c MIL1RAP expression was examined by RT-PCR in 10 pairs of human CC and adjacent non-cancerous tissues. SIL1RAP was detected as a control. d Ms751 cells were infected with either retrovirus expressing mIL1RAP-knockdown shRNA (shmIL1RAP) or control shRNA and selected for puromycin resistance. Stable Ms751 cells were injected into nude mice which were randomly divided into two groups $(n=6)$. Mice were killed at 5 weeks after injection. Tumors were excised from the mice and weighed (scale bar:
$1 \mathrm{~cm})$. e Results are shown as mean \pm s.d. of the tumor weight. $\mathbf{f}$ Timecourse of xenograft growth. Tumor volumes of the mice described in $\mathbf{d}$ were measured every week. Each point represents the mean \pm s.d. of the tumors. g Ms751/shSRSF10 or shRNA control cells were transfected with either retrovirus expressing mIL1RAP or empty-vector control (vector). Stable Ms751 cells were injected into nude mice which were randomly divided into three groups $(n=6$, scale bar: 1 $\mathrm{cm})$. $\mathbf{h}$ Results are shown as mean \pm s.d. of the tumor weight. $\mathbf{i}$ Timecourse of xenograft growth. The tumor volumes of mice described in $\mathbf{g}$ were measured every week. Each point represents the mean \pm s.d. of the tumors. $* P<0.05 ; * * P<0.01 ; * * * P<0.001$

1149 SRSF10-affected AS genes were mainly involved in the gene expression, cancer-related cell cycle and immunity function process (Supplementary Fig. S3d).

Among those AS genes, IL1RAP came into our notice, whose dysregulation was associated with inflammation and the prognosis of hematological malignant tumors and solid tumors $[24,25]$. The splice variant formed by an alternative terminator in exon 10.2 generates the 356-amino-acid-long soluble form of IL1RAP, sIL1RAP, which lacks the transmembrane and intracellular domain, whereas the splice variant derived from terminator in exon 13 encodes the 570- 
a

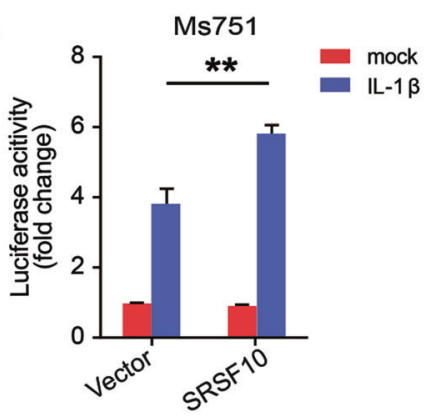

b

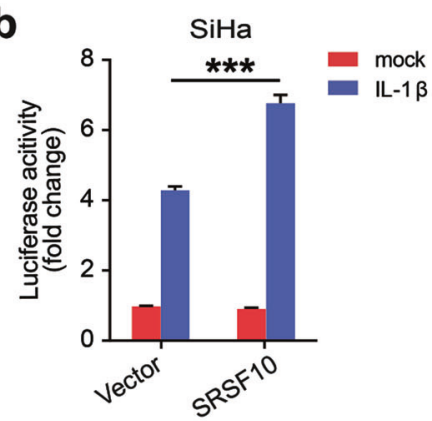

C

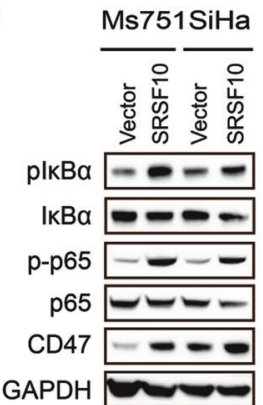

d

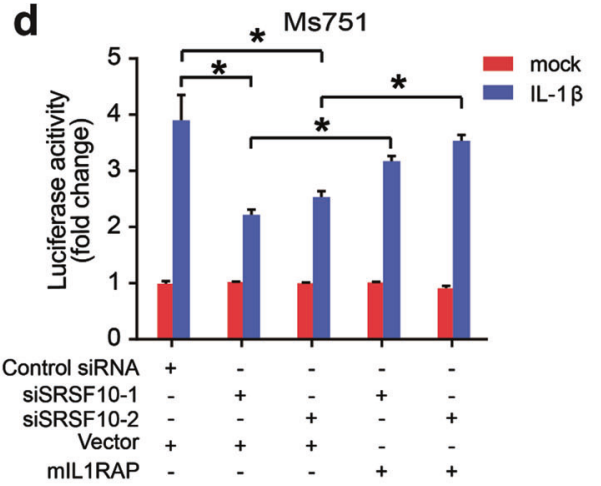

f

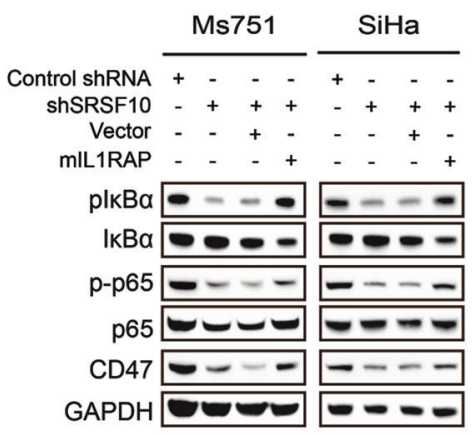

e

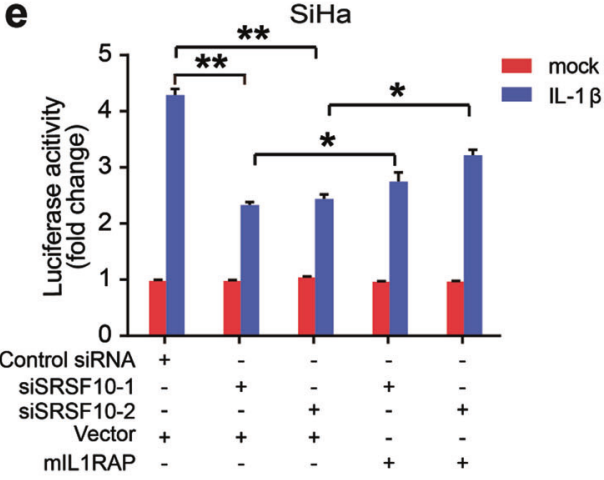

g

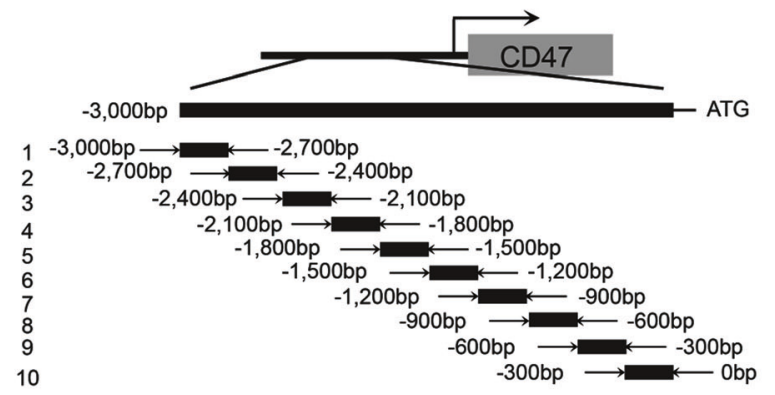

i

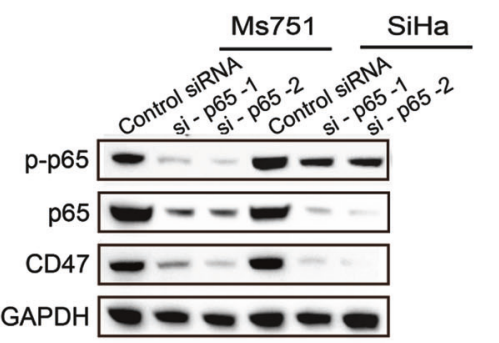

h

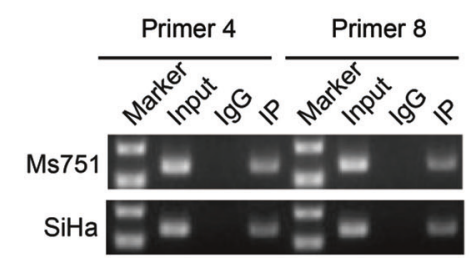

j k

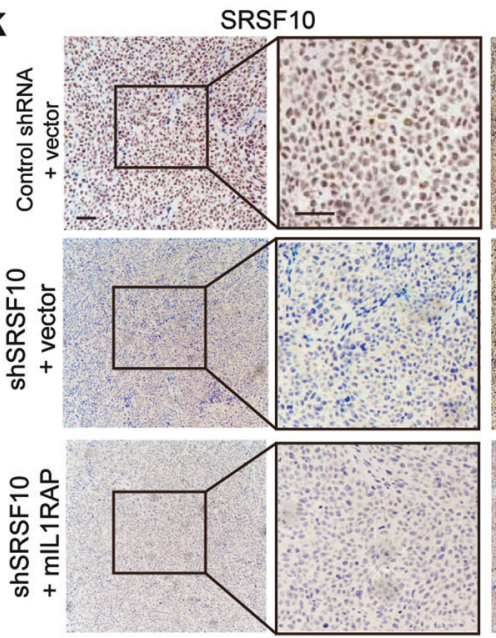

CD47

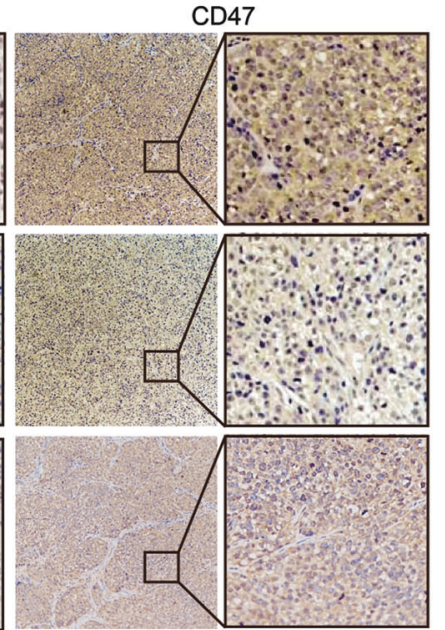

amino-acid membrane form of IL1RAP, mIL1RAP, which forms a complex with interleukin (IL)-1/IL-1RI to initiate IL-1 $\beta$ signal transduction (Supplementary Fig. S4) [26]. The increased alternate terminator (AT) of 13 exon and decreased AT of 10.2 exon were observed in the SRSF10high group compared with the SRSF10-low group (Fig. 4a, b), suggesting that SRSF10 may promote the expression of mIL1RAP while inhibiting the production of sIL1RAP. 
Fig. 6 SRSF10-mediated mIL1RAP upregulates the expression of CD47 via promoting IL-1 $\beta$-induced NF- $\mathrm{KB}$ activation. a, b Identification of SRSF10 as an enhancer of IL- $1 \beta$-induced NF- $\kappa B$ activation by luciferase reporter assay. Ms751 and $\mathrm{SiHa}$ cells were infected with either retrovirus expressing SRSF10 or empty-vector control (vector), then the cells were further transfected with NF- $\mathrm{KB}$ luciferase reporter. A total of $20 \mathrm{~h}$ after transfection, cells were treated with IL-1 $\beta$ (10 ng/ $\mathrm{mL}$ ) or left untreated for $8 \mathrm{~h}$ before reporter assays. c Western blot analysis of $\mathrm{p}-\mathrm{I} \kappa \mathrm{B} \alpha, \mathrm{I} \kappa \mathrm{B} \alpha, \mathrm{p}-\mathrm{NF}-\kappa \mathrm{B}$ p65, NF- $\mathrm{B}$ p65, and CD47 was performed in Ms751 and $\mathrm{SiHa}$ cells indicated in a, b. Cells were treated with IL-1 $\beta(10 \mathrm{ng} / \mathrm{mL})$ for $8 \mathrm{~h}$. d, e Luciferase reporter assay to detect the effect of SRSF10-mediated mIL1RAP on IL-1 $\beta$-induced NF-KB activation. Ms751 and $\mathrm{SiHa}$ cells were infected with either retrovirus expressing mIL1RAP or control vector, then the cells were further transfected with SRSF10-RNAi (siSRSF10-1, siSRSF10-2) or control siRNA for $36 \mathrm{~h}$ before NF- $\mathrm{\kappa B}$ luciferase reporter transfection. Cells were treated with IL- $1 \beta$ indicated in a. f Western blot analysis for NF- $\kappa \mathrm{B}$ activation and CD47 expression was performed in Ms751 and SiHa cells indicated in Fig. 5g and Supplementary Fig. S6d. Cells were treated with IL-1 $\beta(10 \mathrm{ng} / \mathrm{mL})$ for $8 \mathrm{~h}$. $\mathbf{g}$ The sketch map of primers for CD47 promoter sequences. Ten primer sets with a 300-bp partition were designed for PCR to test the direct binding of p65 to the CD47 promoter and the primer pairs produced 10 fragments of $300 \mathrm{bp}$. h The chromatin DNA of Ms751 and SiHa cells was chromatinimmunoprecipitated (ChIP) with p65 antibody. Sonicated input DNA and IgG was used as control. Amplification of the CD47 promoter sequence from ChIP DNA validated the binding of p65 to CD47 promoter site 4 and 8. i Western blot analysis was performed to detect the regulation of p65-knockdown on CD47 expression. Ms751 and SiHa cells were transfected with p65-RNAi or control siRNA. j NF- $\kappa$ B activation upregulated the expression of CD47 as detected by western blot. IL-1 $\beta$ was used as a stimulator of NF-kB. Ms751 and SiHa cells were treated with IL-1 $\beta$ as indicated in a. k IHC staining of SRSF10 and CD47 in tumors from Ms751 cell xenograft nude mice indicated in Fig. $5 \mathrm{~g}$ (scale bar: $200 \mu \mathrm{m}$ ). $* P<0.05 ; * * P<0.01 ; * * * P<0.001$

As an initial test for verification, we examined the effects of SRSF10 knockdown on the splicing of IL1RAP in CC cells by RNA-seq analysis. There was a exclusion of exon 13 after SRSF10 knockdown, whereas no remakable changes were observed in exon 10.2 (Fig. 4c, d). This results indicate that the inclusion of exon 13 was regulated by SRSF10, whereas the regulation of SRSF10 on exon 10.2 might be slight. Consistently, further RT-PCR confirmed that the expression of mIL1RAP was significantly downregulated after SRSF10 knockdown, whereas there were no significant differences in the changes in SIL1RAP expression (Fig. 4e, f). In addition, there was a positive correlation between SRSF10 and mIL1RAP expression, while no correlation was observed between SRSF10 and sIL1RAP in clinical CC samples (Fig. 4g-j). Thus, we verified that SRSF10 is a main splicing regulator of mIL1RAP in CC. Next, we tried to demonstrate whether SRSF10 facilitate the production of mIL1RAP in a positiondependent manner by directly binding to IL1RAP exon 13 . After transiently overexpressing hemagglutinin (HA)-tagged SRSF10 complementary DNA in SiHa cells, in vitro CLIP-PCR analysis was performed with primer pairs specific for the alternative exon 13 of IL1RAP. As shown in Fig. 4k, SRSF10 bound to exon 13 compared with the empty-vector control, consistent with the reported positiondependent splicing regulation effect of SRSF10. Taken together, mIL1RAP is a direct splicing target regulated by SRSF10.

\section{Dysregulated mIL1RAP participates in the tumorigenic potential of SRSF10 in CC}

As SRSF10 promotes the production of mIL1RAP, we next evaluated whether the expression of mIL1RAP is upregulated in CC. In silico analysis of GEO and Oncomine data sets showed that the expression of mIL1RAP was increased in CC tissues compared with normal tissues (Fig. 5a, b and Supplementary Fig. S5a-c). Further RT-PCR verification revealed a consistent increase of mIL1RAP expression in 10 $\mathrm{CC}$ tissues compared to their corresponding adjacent noncancerous tissues (Fig. 5c). In addition, mIL1RAP expression was much higher in HPV16/18-positive CCs than in HPV-negative ones (Supplementary Fig. S5d). Hence, these observations suggest that mIL1RAP overexpression might be involved in CC development. Accordingly, the role of mIL1RAP in CC was then examined using Ms751 and SiHa xenografts in nude mice by designing a shRNA targeted against the $\mathrm{C}$ terminus for isoform-specific knockdown of mIL1RAP (Fig. 5d and Supplementary Fig. S6a). Knockdown of mIL1RAP caused a significant decrease in tumor growth rate and tumor weight (Fig. 5e, f and Supplementary Fig. S6b, c). Overall, these data infer that dysregulated mIL1RAP contributes to the development of CC.

As our experiments demonstrated mIL1RAP was a direct target of SRSF10, we next asked whether mIL1RAP participates in the oncogenic role of SRSF10 in CC. To this end, mIL1RAP was stably induced into SRSF10knockdown CC cells to observe whether re-expression of mIL1RAP could rescue the reduced tumorigenesis of SRSF10-knockdown cells in vivo. Exhilaratingly, overexpression of mIL1RAP significantly reduced the inhibitory effects of shSRSF10 on CC growth (Fig. $5 \mathrm{~g}-\mathrm{i}$ and Supplementary Fig. S6d-f), underscoring the cancerigenic function of SRSF10-mediated mIL1RAP in CC.

\section{SRSF10-mediated mIL1RAP-NF-KB axis upregulates the "don't eat me" signal CD47 in CC}

IL1RAP is indispensable for IL-1 $\beta$ signal transduction and IL-1 $\beta$ level is increased in CC (Supplementary Fig. S7) [27]. IL-1 $\beta$ strongly elicit activation of transcription factor $\mathrm{NF}-\mathrm{kB}[28,29]$, which has a pivotal role in driving the progression of inflammation to cancer [30, 31]. Thus, we logically tested whether SRSF10-mediated mIL1RAP promote $\mathrm{CC}$ progression by regulating IL- $1 \beta$-induced NF- $\mathrm{KB}$ activation. As predicted, the luciferase reporter assays showed that overexpression of SRSF10 potentiated IL-1 $\beta$ - 

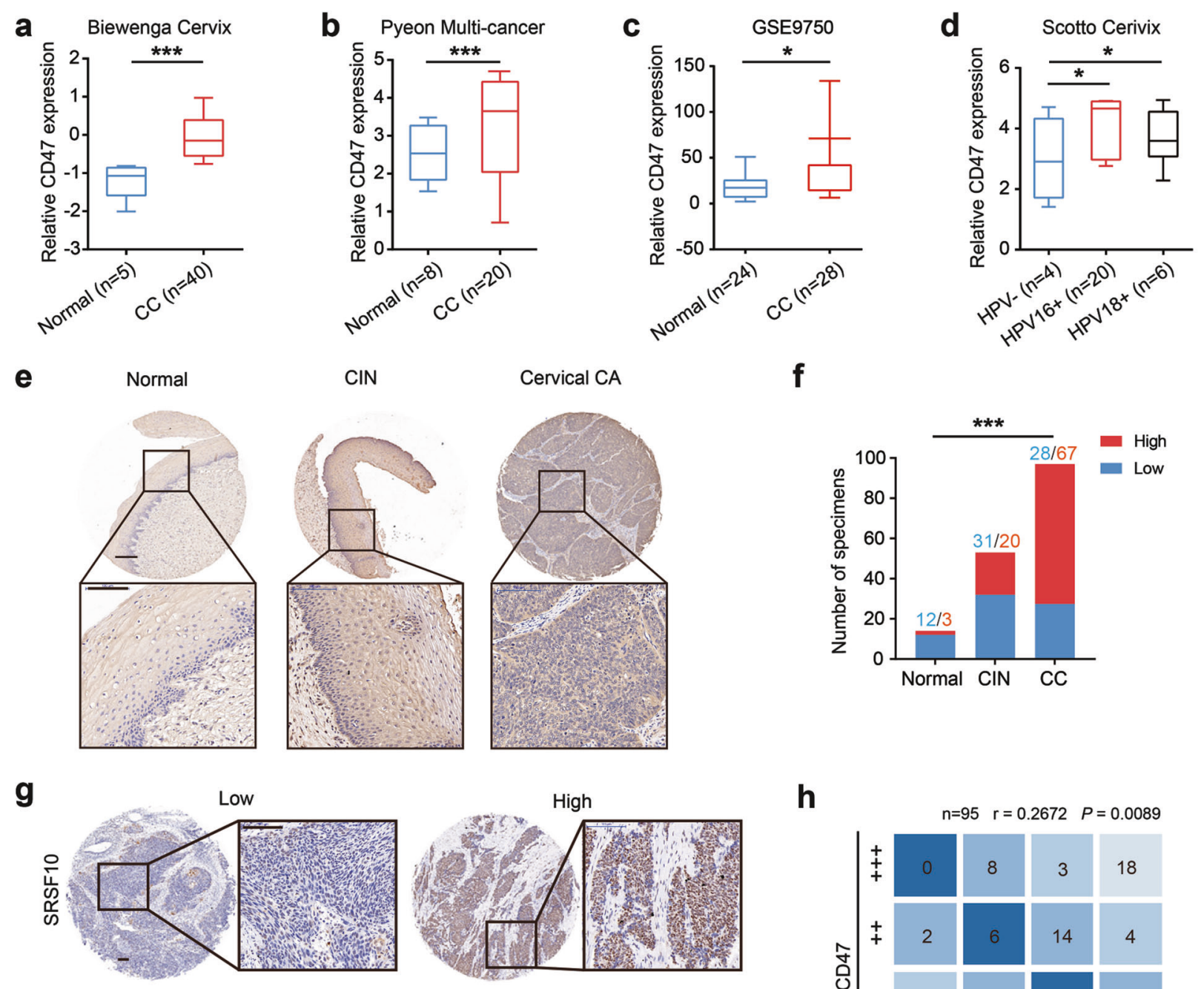

h
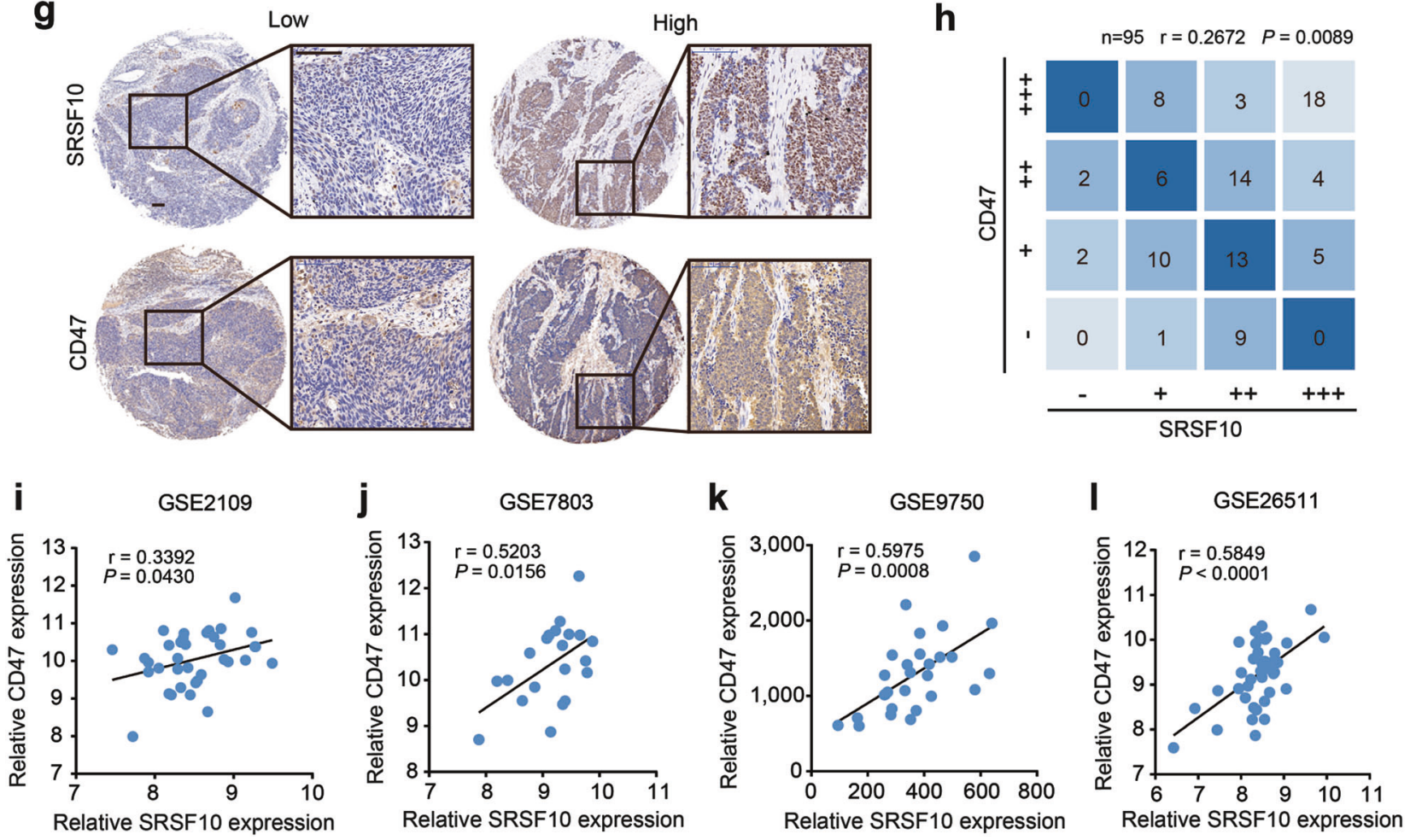

induced NF- $\mathrm{KB}$ activation, whereas knockdown of SRSF10 markedly inhibited IL-1 $\beta$-induced NF- $\mathrm{KB}$ activation (Fig. $6 \mathrm{a}, \mathrm{b}, \mathrm{d}, \mathrm{e})$. More intriguingly, overexpression of mIL1RAP could rescue the diminished NF- $\mathrm{KB}$ activation of SRSF10- knockdown cells (Fig. 6d, e). Consistently, overexpression of SRSF10 increased the phosphorylation of IkB $\alpha$ and p65, whereas SRSF10-knockdown repressed the the expressions of $\mathrm{p}-\mathrm{I} \mathrm{K} \mathrm{B} \alpha$ and $\mathrm{p}-\mathrm{p} 65$, which were rescued by re-expression 
Fig. 7 Increased CD47 parallels SRSF10 expression in CC. Analyses of CD47 mRNA levels in normal cervical tissues and CC in the published a Biewenga Cervix data set, b Pyeon Multi-cancer data set and $\mathbf{c}$ GSE9750. d Box plots comparing CD47 mRNA levels in HPVnegative CCs and HPV 16/18-positive CCs in the published Scotto Cervix dataset from Oncomine. e Representative photographs of CD47 immunoreactivity in normal tissue, CIN, and CCs in TMA (scale bar: $200 \mu \mathrm{m})$. f The constituent ratio of CD47 expression assessed by blinded IHC analyses in normal tissues, CINs and CCs indicated in Fig. 2e. g Representative photographs of the SRSF10 and CD47 immunoreactivity in the same samples (scale bar: $200 \mu \mathrm{m}$ ). h The correlation of CD47 and SRSF10 protein levels in CC. $\mathbf{i}-\mathbf{l}$ A positive correlation between the expression levels of CD47 and SRSF10 was observed in four independent $\mathrm{CC}$ data sets. $* P<0.05$; $* * P<0.01$; $* * * P<0.001$

of mIL1RAP (Fig. 6c, f). Together, these results indicate that SRSF10-mediated mIL1RAP promotes the IL-1 $\beta$ induced NF- $\kappa \mathrm{B}$ activation in $\mathrm{CC}$.

As cancer immunity is involved in SRSF10-regulated tumorigenesis (Supplementary Fig. S3d), we further explored whether SRSF10-mediated mIL1RAP-NF- $\kappa B$ signaling contributes to $\mathrm{CC}$ progression by regulating the expression of immune molecules. After a primary prediction using the JASPAR websites, we focused on CD47 as a potential target of NF- $\mathrm{KB}$ p65. CD47 is a cell surface glycoprotein that inhibits phagocytosis by binding to signal regulatory protein alpha on macrophages. In this way, CD47 serves as a "don't eat me" signal. Thus, when CD47 protein is expressed on the surface of solid tumor cells, it serves as a potent signal to escape from immune surveillance. Therefore, blocking CD47 with antibody turns off "don't eat me" signal and favors phagocytosis, being expected to be an efficacious treatment for tumors [32]. The ChIP-PCR confirmed that p65 could bind to CD47 promoter site 4 and 8 in both Ms751 and SiHa cells (Fig. 6g, h). To verify the regulatory role of p65 on CD47 expression, siRNAs targeted against p65 were used. As shown in Fig. 6i, p65-knockdown significantly downregulated CD47 expression in $\mathrm{CC}$ cells. Conversely, after activating p65 upon treatment of $\mathrm{CC}$ cells with the NF- $\mathrm{\kappa B}$ stimulator, IL$1 \beta$, the expression of CD47 was upregulated (Fig. 6j). Collectively, these results demonstrate the direct transcriptional regulatory role of p65 on CD47.

Furthermore, SRSF10 knockdown caused a significant decrease in CD47 expression in both CC cells, but overexpression of mIL1RAP could rescue the diminished CD47 expression (Fig. 6f, k). Inversely, the expression of CD47 was upregulated after SRSF10 overexpression (Fig. 6c). In vitro phagocytosis assays also showed that phagocytosis was increased in SRSF10-knockdown cells, which was rescued by re-expression of mIL1RAP. Conversely, overexpression of SRSF10 decreased the phagocytosis of macrophages (Supplementary Fig. S8). Hence, these observation imply that SRSF10mediated mIL1RAP upregulate the "don't eat me" signal
CD47 via promoting IL- $1 \beta$-induced $N F-\kappa B$ activation to in $\mathrm{CC}$.

\section{The expression of CD47 is significantly upregulated and positively correlated to SRSF10 in clinical CC samples}

We next asked whether CD47 expression is upregulated in clinical CC samples. The Oncomine and GSE9750 data sets showed a remarkable increase in CD47 mRNA levels in CC tissues compared with normal tissues (Fig. 7a-c). The upregulation of CD47 was associated with HPV16/18 infection (Fig. 7d). To provide more evidence, the protein level of CD47 was examined in the above CC tissue array by IHC. As shown in Figs. 7e, f, the expression levels of CD47 were increased with the malignant transformation from normal cervical epithelia to CINs to CCs. Significantly, a positive correlation was observed between the expression of SRSF10 and CD47 (Fig. 7g, h). A similar correlation was also observed at the mRNA level between SRSF10 and CD47 in clinical CC samples (Fig. 7i-1). To sum up, these results indicate that SRSF10 promotes CC tumorigenesis partly through modulating the mIL1RAPNF- $\kappa \mathrm{B}$ axis to upregulate the "don't eat me" signal CD47.

\section{Discussion}

In this study, we identified SRSF10, a splicing factor, is significantly increased and necessary for the tumorigenic capacity of HPV16/18-positive CC. High-risk human papillomavirus (HR-HPV) viral oncoproteins E6E7 activate the expression of SRSF10 via the transcription factor E2F1. SRSF10 facilitates the production of mIL1RAP by modulating AS of IL1RAP exon 13. SRSF10-mediated mIL1RAP promotes the IL- $1 \beta$-induced $N F-\kappa B$ activation, which initiates the transcription of the "don't eat me" signal CD47 to avoid elimination by macrophage phagocytosis. There was a significant correlation among SRSF10, mIL1RAP and CD47 expression in clinical CC tissues, further supporting the regulatory axis of SRSF10, mIL1RAP and CD47. Thus, this study shows the close relationship among $\mathrm{AS}$, inflammatory molecules and immune surveillance in HPV16/18-positive tumorigenesis (Fig. 8).

HR-HPV E6E7 oncoproteins are considered the major etiologic contributor to $\mathrm{CC}$ carcinogenesis, but they are not sufficient for full malignant transformation. Other risk elements, such as hormones, the environment, and immunology, have also been considered [33, 34]. However, aberrant AS, which elicits control over the major hallmarks of cancer [8, 12], has not been explored in CC. Do E6 and E7 influence AS? Does AS act as an "accomplice" of E6E7 in $\mathrm{CC}$ progression? Hence, we explored the oncogenic effects 

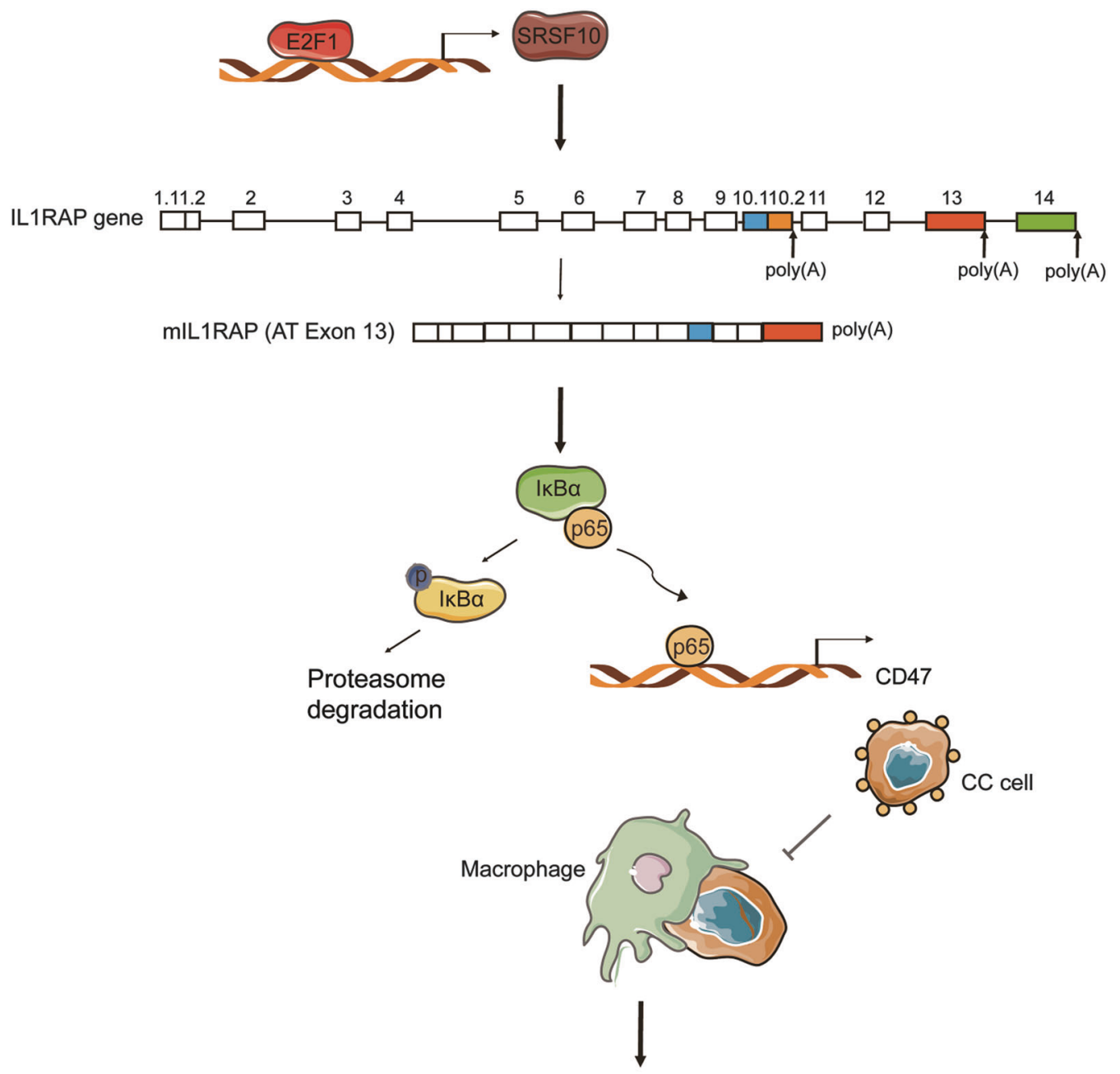

CC development

Fig. 8 Model of the SRSF10-regulated mIL1RAP-NF-кB axis upregulating the "don't eat me" signal CD47 in HPV16/18-positive CC

of E6E7 from the perspective of AS. Microarray and bioinformatics analyses have provided strong evidence that E6E7 regulates the expression of splicing factors and the most remarkable changed splicing factor is SRSF10. SRSF10 was transcriptionally activated via the E6E7-E2F1 axis, and knockdown of SRSF10 demonstrated its essential role in CC tumorigenesis. SRSF10 is a splicing regulator of the SR protein family, which can function both positively and negatively in regulating exon inclusion depending on its binding locations with the pre-mRNA [22, 23, 35]. Previous studies have shown that SRSF10 is upregulated in colorectal cancer as a key regulator of BCLAF1 pre-mRNA splicing and thus maintaining the oncogenic phenotype of colon cancer cells $[36,37]$. In addition, the expression and distribution of SRSF10 are developmentally and hormonally regulated and increased in endometrial carcinomas as well as ovarian cancers, supporting its potential involvement in uterine development and tumorigenesis [38]. Here, our study infered the oncogenic role of SRSF10.
$\mathrm{CC}$ is an inflammation-related tumor and inflammation could be considered a co-factor in CC progression by promoting cell proliferation and metastasis, inhibiting apoptosis and secreting immune suppressors [39]. The interplay of viral oncoproteins and inflammatory factors may lead to the development of persistent infection by continuous immune evasion, which promotes lesion progression and ultimately malignancy [40]. Cancer cells can directly produce IL-1 $\beta$, and the IL-1 $\beta-N F-\kappa B$ inflammatory pathway promotes the progression of multiple tumor types [41]. The level of IL-1 $\beta$ is upregulated in CC and IL1RAP is indispensable for the transmission of IL-1 $\beta-\mathrm{NF}-\kappa \mathrm{B}$ inflammation signaling [42]. IL1RAP is associated with the prognosis of myeloid leukemia and head and neck squamous cell carcinoma patients and antibodies targeting IL1RAP showed therapeutic effects in xenograft models of myeloid leukemia [43]. Unfortunately, the splicing regulation mechanism of IL1RAP remains little-understood. Here, we identified AS of IL1RAP exon 13 as a direct SRSF10 target via 
transcriptome analysis as well as CLIP and PCR validation. Consistent with SRSF10, mIL1RAP was upregulated and promoted the carcinogenesis capacity of CC. However, the splicing regulation on IL1RAP exon 10.2 is slight, which emphasizes the complicacy of AS regulation in vivo. Most AS events are likely regulated by multiple splicing factors acting synergistically or antagonistically [5]. Thus, from the splicing regulation of SRSF10 on IL1RAP, our study revealed the function of mIL1RAP in CC and the interaction between AS and inflammatory pathways in cancer.

$\mathrm{NF}-\mathrm{\kappa B}$ activation was identified as a key modulator in driving inflammation to cancer [44], and many studies have reported that NF- $\mathrm{KB}$ is a major factor promoting the malignant conversion and progression of CC cells $[45,46]$. Proverbially, the malignant features of cancer cells can not be manifested without an important interplay between cancer cells and their local environment [47]. Thus, in the clinical setting the manipulation of the tumor microenvironment to encapsulate or destroy cancer cells could be used as an approach to prevent as well as treat cancer [48]. Here, from a tumor microenvironment perspective, we found that SRSF10-mIL1RAP axis promotes IL-1 $\beta$-induced $\mathrm{NF}-\mathrm{\kappa B}$ activation to upregulate the "don't eat me" signal CD47, thus avoiding macrophage phagocytosis. Macrophages act as corrupted policemen and lead to immune escape. Recent studies showed that NF-KB directly regulates CD47 expression in hepatocellular cancer, further supporting our findings [49]. Together, these results indicate that NF- $\kappa B$ provides a mechanistic link between SRSF10-mediated mIL1RAP splicing and cancer immune microenvironment.

The study suggests the cancer-promoting function and the therapeutic potential of mIL1RAP and CD47 in CC. Encouragingly, in vivo administration of IL1RAP or CD47 antibodies in mice transplanted with tumor cells has provided therapeutic effects [32] and the clinical trials for intratumoral injections of anti-CD47 drug TTI-621 and chimeric antigen receptor T-cell immunotherapy targeting IL1RAP are recruiting (NCT02890368, NCT02842320), so it will also be interesting to explore and develop an mIL1RAP isoform-specific blocker or CD47 targeted therapy for CC. Besides, our study only shows an example of mechanistic link among HPV infection, alternative splicing and CC. Beyond the involvement of the mIL1RAP-NF- $\mathrm{KB}-$ CD47 axis, other SRSF10-regulated splicing targets can also be implicated in the cancer-promoting function of SRSF10. Future studies are required to investigate the roles of these splice isoforms in CC development. Certainly, SRSF10 is only a representation of numerous splicing factors, the biological functions and clinical values of which merit further investigations. AS orchestrates interactions between various types of proteins and between proteins and nucleic acids. Evaluation of AS events in tumor can be used to identify novel disease markers and drug-sensitive targets to overcome the limits of the small molecule inhibitors currently used for treating patients with tumor [50]. The use of aberrant splicing as disease markers has been reported, however, little is known about the use of splicing abnormalities as drug targets in tumor. Therefore, to develop a potential therapeutic approaches that can be used to target splicing abnormalities or splicing regulators is the direction of our endeavor.

In summary, we have demonstrated the tumorigenic roles of SRSF10 and mIL1RAP by promoting IL- $1 \beta$-induced NF$\kappa \mathrm{B}$ activation to upregulate the expression of CD47. Consequently, our study reveals the close relationship among HPV infection, alternative splicing and tumor microenvironment, and it establishes SRSF10-mIL1RAP-CD47 as a potential therapeutic target for $\mathrm{CC}$.

\section{Materials and methods}

\section{Cell culture and transfection}

Human cervical cancer MS751, SiHa, and embryonic kidney $293 \mathrm{~T}$ cell lines were cultured in Dulbecco's modified Eagle's medium containing 10\% fetal bovine serum (FBS) and $1 \%$ penicillin/streptomycin $(\mathrm{P} / \mathrm{S})$. Human cervical cancer Caski and promonocytic THP1 cell lines were cultured in RPMI 1640 containing 10\% FBS, 2 mM glutamine and $1 \% \mathrm{P} / \mathrm{S}$. All cells were incubated at $37^{\circ} \mathrm{C}$ in a humidified atmosphere containing $5 \% \mathrm{CO}_{2}$.

SiRNA targeting against different genes were purchased from Gene Pharma (Shanghai, China) and the sequences are listed in Supplementary Table S2, respectively. SiRNA transfection was performed using LipofectamineRNAiMAX (13778150, Invitrogen, Carlsbad, CA, USA). ShSRSF10 and shmIL1RAP retroviruses were generated with PLKO-puro lentiviral vectors in 293 T cells. Lentivirusexpressing SRSF10 or mIL1RAP was generated with pCDH-neo lentiviral vectors in 293T cells. Ms751 and SiHa cells were infected with lentivirus and selected for puromycin or neomycin resistance.

\section{Human CC samples and tissue microarray}

Ten pairs of primary $\mathrm{CC}$ and their corresponding adjacent non-cancerous tissues were obtained from CC patients treated at Shanghai Jiao Tong Affiliated Sixth People's Hospital after receiving their informed consent. Fresh samples were snap-frozen in liquid nitrogen and used for RT-PCR analysis. Tissue microarrays containing 15 normal cervical epithelia, $51 \mathrm{CINs}$ and $95 \mathrm{CC}$ were purchased from Superbiotek (Shanghai, China), used for immunohistochemistry (IHC) and IHC score analysis. 


\section{Quantitative real-time PCR (qPCR)}

Total RNAs were extracted using RNAiso reagent (9109, Takara, Dalian, China). Reversed-transcription was performed as described [51]. All primers are listed in Supplementary Table S3.

\section{Western blot}

Western blot analysis was performed as described [51]. Antibodies used were mouse anti-GAPDH (1:10000, ab8245), anti-SRSF10 (1:1000, ab77209), rabbit anti-E2F1 (1:1000, ab179445), anti-CD47 (1:1000, ab175388) from Abcam (Cambridge, UK), anti-IкB $\alpha(1: 1000,4814)$, antiPhospho-IкB $\alpha$ (1:1000, 9246), anti-NF-кB p65 (1:1000, 8242), anti-Phospho-NF-кB p65 (1:1000, 3033) from Cell Signaling Technology (Danvers, MA, USA).

\section{Immunohistochemistry}

Immunohistochemistry (IHC) was performed as previously reported [51]. Anti-SRSF10 (1:200, GTX47232, GeneTex, San Antonio, USA) or anti-CD47 (1:100, ab175388, Abcam) antibodies were used. Each tumor was assigned with a score according to the intensity of the nuclei or cytoplasm staining $(0=$ no staining, $1=$ weak staining, $2=$ moderate staining, and $3=$ strong staining) and the proportion of stained tumor cells $(0=0 \%, 1=1-10 \%, 2=$ $11-50 \%, 3=51-80 \%$, and $4=81-100 \%$ ), as judged independently by two pathologists in a blinded manner. The final immunoreactive score was determined by multiplying the intensity scores by the extent of positivity scores of stained cells, with "-" for a score of 0, " + " for a score of $1-4$, " ++ " for a score of 5-8 and " +++ " for a score of 9-12. Tumors with scores $\geq 5$ were classified into the high expression group, whereas the others were classified into the low expression group.

\section{ChIP and RNA immunoprecipitation}

ChIP assays were performed as previously reported [52]. Antibodies against E2F1 (1:50, ab179445, Abcam) and NFкB p65 (1:1000, ab19870, Abcam) were used for IP. The primers used for the ChIP assay are listed in Supplementary Table S4.

HA-tagged SRSF10 plasmid or empty vector was transiently transfected into MS751 cells, and IP was performed with anti-HA antibody $(5 \mu \mathrm{g}$ per reaction, SAB1411733, Sigma-Aldrich)-coupled protein A/G magnetic beads ( $50 \mu \mathrm{g}$ per reaction) using the Magna RIP kit (Millipore). RNA was extracted from the immunoprecipitate and reversetranscribed into cDNA for PCR detection. RNA enrichment was measured by RT-PCR with primers specific for
IL1RAP exon13. The details of the primer sequences are provided in Supplementary Table S5.

\section{Luciferase reporter assay}

Luciferase reporter plasmids containing wild-type and mutant SRSF10 promoters were constructed in the pGL3B plasmid and verified for the dual luciferase reporter assay. $\mathrm{NF}-\mathrm{\kappa B}$ luciferase reporter plasmid was purchased from genomeditech (Shanghai, China). Cells were lysed $48 \mathrm{~h}$ post transfection with passive lysis buffer using the dual luciferase assay kit (E1910, E1960, Promega, Madison, WI, USA). Luciferase activity was tested with a luminometer. The results are expressed as the ration of the luciferase value to the Renilla value.

\section{Phagocytosis assay}

THP1 cells were differentiated into macrophages by incubation with PMA (100 ng/mL, P1585, Sigma-Aldrich) for $48 \mathrm{~h}$ and then plated $\left(2 \times 10^{5}\right.$ per well $)$ in a six-well tissueculture plate $24 \mathrm{~h}$ before the experiment. For in vitro phagocytosis assay, similarly to previously described [53, 54], macrophages were labled with $1 \mu \mathrm{M}$ Cell Proliferation Dye eFluor 670 (65-0840, ThermoFisher Scientific, Carlsbad, CA, USA) and CC cells were stained with $0.25 \mu \mathrm{M}$ carboxyfluorescein succinimidyl ester (CFSE) (C34570, ThermoFisher Scientific, Carlsbad, CA, USA) according to the manufacturer's protocol. Macrophages were incubated in serum-free medium for $2 \mathrm{~h}$ before adding $8 \times 10^{5} \mathrm{CFSE}$ labeled CC cells. After co-culture for $3 \mathrm{~h}$ at $37^{\circ} \mathrm{C}$, the cells were harvested and flow cytometry (LSRFortessa, BD Biosciences) was performed. A total of $2 \times 10^{5}$ cells in each sample were analyzed. Unstained control and single stained cells were prepared for gating. The phagocytosis was calculated as the percentage of macrophages engulfing tumor cells $[670+\mathrm{CFSE}+$ cells (Q2)] among total macrophages $[670+$ cells $(\mathrm{Q} 1+\mathrm{Q} 2)]$ and normalized to the result obtained from the control shRNA+vector group.

\section{Xenograft assays}

Xenograft model was built as previously described [55]. All mice were killed at day 35 , and the xenograft was stripped out and weighed for further analysis.

\section{Microarray experiment and RNA-seq}

Microarray experiment were performed as previously described [15]. For RNA-seq, total RNAs isolated from Ms751 and $\mathrm{SiHa}$ cells transfected with siSRSF10 or control siRNA were subjected to RNA-seq using Illumia Hiseq 2000 system. Reads were mapped 
to a reference human genome and transcriptome using Tophat.

\section{TCGA spliceseq data analysis}

According to the SRSF10 expression, 40 TCGA CC samples were divided into the high SRSF10 expression group $(n=20)$ and the low SRSF10 expression group $(n=20)$ to explore the AS events related to SRSF10 in CC. To quantify the possible SRSF10-related splice events, the percent spliced in index (PSI) was calculated [21]. PSI is the ratio of normalized read counts indicating inclusion of a transcript element over the total normalized reads for that event (both inclusion and exclusion reads). The PSI data were downloaded from the TCGA spliceseq database (http:// bioinformatics.mdanderson.org/TCGASpliceSeq/) and the differential AS events related to SRSF10 in CC were screened with threshold values: $\mid \Delta$ PSI $\mid \geq 0.2$.

\section{Statistics}

Statistical analyses and graphical representations were performed using SPSS 20.0 (Chicago, USA) and GraphPad Prism 7 (San Diego, USA) software. All data presented as histograms refer to the mean \pm s.d. of the total number of independent experiments. Statistical analysis was performed using the Student's $t$-test for two groups or ANOVA for multiple groups. Spearman rank correlation test was used to analyze the correlation between two parameters. $P<0.05$ was considered statistically significant.

Acknowledgements We thank Dr. Xueli Zhang (State Key Laboratory for Oncogenes and Related Genes, Shanghai, China) for critical reading of manuscript; Experimental Animal Center of East China Normal University for animal feeding. This work was supported by grants from the National Natural Science Foundation of China (81372794; 81672565; 81502382), and the Science and Technology Commission of Shanghai Municipality-Key Basic Research Foundation (13JC404502).

Author contributions YCT, ZZZ, and FL designed the research. FL, MD, QYX, and SHJ conceived and performed the experiments. SHJ, YHW, JL, LPH, YLZ and SJZ provided research materials and methods. XLZ, ZHA and LM provided fresh clinical specimens. QY and YLZ performed bioinformatics analysis. FL wrote the manuscript. All authors read and approved the final manuscript.

\section{Compliance with ethical standards}

Conflict of interest The authors declare that they have no competing interests.

Open Access This article is licensed under a Creative Commons Attribution-NonCommercial-ShareAlike 4.0 International License, which permits any non-commercial use, sharing, adaptation, distribution and reproduction in any medium or format, as long as you give appropriate credit to the original author(s) and the source, provide a link to the Creative Commons license, and indicate if changes were made. If you remix, transform, or build upon this article or a part thereof, you must distribute your contributions under the same license as the original. The images or other third party material in this article are included in the article's Creative Commons license, unless indicated otherwise in a credit line to the material. If material is not included in the article's Creative Commons license and your intended use is not permitted by statutory regulation or exceeds the permitted use, you will need to obtain permission directly from the copyright holder. To view a copy of this license, visit http://creativecommons. org/licenses/by-nc-sa/4.0/.

\section{References}

1. Wang ET, Sandberg R, Luo S, Khrebtukova I, Zhang L, Mayr C, et al. Alternative isoform regulation in human tissue transcriptomes. Nature. 2008;456:470-476.

2. Keren H, Lev-Maor G, Ast G. Alternative splicing and evolution: diversification, exon definition and function. Nat Rev Genet. 2010;11:345-355.

3. Venables JP, Klinck R, Koh C, Gervais-Bird J, Bramard A, Inkel $\mathrm{L}$, et al. Cancer-associated regulation of alternative splicing. Nat Struct Mol Biol. 2009;16:670-676.

4. Kozlovski I, Siegfried Z, Amar-Schwartz A, Karni R. The role of RNA alternative splicing in regulating cancer metabolism. Hum Genet. 2017;136:1113-127.

5. Tsai YS, Dominguez D, Gomez SM, Wang Z. Transcriptomewide identification and study of cancer-specific splicing events across multiple tumors. Oncotarget. 2015;6:6825-6839.

6. Matera AG, Wang Z. A day in the life of the spliceosome. Nat Rev Mol Cell Biol. 2014;15:108-121.

7. Munkley J, Livermore K, Rajan P, Elliott DJ. RNA splicing and splicing regulator changes in prostate cancer pathology. Hum Genet. 2017;136:1143-54.

8. Oltean S, Bates DO. Hallmarks of alternative splicing in cancer. Oncogene. 2014;33:5311-538.

9. Centers for Disease Control and Prevention. Global Cancer Statistics. http://www.cdc.gov/cancer/international/statistics.htm. Accessed 13 May 2016.

10. World Health Organization. Human papillomavirus (HPV) and cervical cancer. http://www.who.int/mediacentre/factsheets/fs380/ en/. Accessed 10 June 2016.

11. Waggoner SE, Chernicky CL Molecular biology of cervical and vulvar carcinoma. In: Gershenson DM, McGuire WP, Gore M, Quinn MA, Thomas G, eds. Gynaecologic cancer: controversies in management. Philadelphia: Churchill Livingstone; 2004:65-78

12. Ghittoni R, Accardi R, Hasan U, Gheit T, Sylla B, Tommasino M. The biological properties of E6 and E7 oncoproteins from human papillomaviruses. Virus Genes. 2010;40:1-13.

13. Tommasino M, Accardi R, Caldeira S, Dong W, Malanchi I, Smet A, et al. The role of TP53 in cervical carcinogenesis. Hum Mutat. 2003;21:307-12.

14. Walboomers JMM, Jacobs MV, Manos MM, Bosch FX, Kummer $\mathrm{JA}$, Shah KV, et al. Human papillomavirus is a necessary cause of invasive cervical cancer worldwide. J Pathol. 1999;189:12-19.

15. Zhang R, Lu H, Lyu YY, Yang XM, Zhu LY, Yang GD, et al. E6/ E7-P53-POU2F1-CTHRC1 axis promotes cervical cancer metastasis and activates Wnt/PCP pathway. Sci Rep. 2017;7:44744.

16. Hsu TYT, Simon LM, Neill NJ, Marcotte R, Sayad A, Bland CS, et al. The spliceosome is a therapeutic vulnerability in MYCdriven cancer. Nature. 2015;525:384.

17. Zhai Y, Kuick R, Nan B, Ota I, Weiss SJ, Trimble CL, et al. Gene expression analysis of preinvasive and invasive cervical squamous cell carcinomas identifies HOXC10 as a key mediator of invasion. Cancer Res. 2007;67:10163-10172. 
18. Biewenga P, Buist MR, Moerland PD, van Thernaat EVL, van Kampen AHC, ten Kate FJW, et al. Gene expression in early stage cervical cancer. Gynecol Oncol. 2008;108:520-526.

19. Scotto L, Narayan G, Nandula S, Arias-Pulido H, Subramaniyam $\mathrm{S}$, Schneider $\mathrm{A}$, et al. Identification of copy number gain and overexpressed genes on chromosome arm $20 \mathrm{q}$ by an integrative genomic approach in cervical cancer: potential role in progression. Genes Chromosomes Canc. 2008;47:755-765.

20. Pyeon D, Newton NA, Lambert PF, den Boon JA, Sengupta S, Marsit CJ, et al. Fundamental differences in cell cycle deregulation in human papillomavirus-positive and human papillomavirusnegative head/neck and cervical cancers. Cancer Res. 2007;67:4605-4619.

21. Ryan M, Wong WC, Brown R, Akbani R, Su XP, Broom B, et al. TCGASpliceSeq a compendium of alternative mRNA splicing in cancer. Nucleic Acids Res. 2016;44:D1018-D1022.

22. Feng Y, Chen M, Manley JL. Phosphorylation switches the general splicing repressor SRp38 to a sequence-specific activator. Nat Struct Mol Biol. 2008;15:1040-1048.

23. Zhou XX, Wu WW, Li H, Cheng YM, Wei N, Zong J, et al. Transcriptome analysis of alternative splicing events regulated by SRSF10 reveals position-dependent splicing modulation. Nucleic Acids Res. 2014;42:4019-4030.

24. Jensen LE, Whitehead AS. Expression of alternatively spliced interleukin-1receptor accessory protein mRNAs is differentially regulated during inflammation and apoptosis. Cell Signal. 2003;15:793-802.

25. Landberg N, Hansen N, Askmyr M, Agerstam H, Lassen C, Rissler M, et al. IL1RAP expression as a measure of leukemic stem cell burden at diagnosis of chronic myeloid leukemia predicts therapy outcome. Leukemia. 2016;30:253-257.

26. Jensen LE, Muzio M, Mantovani A, Whitehead AS. IL-1 signaling cascade in liver cells and the involvement of a soluble form of the IL-1 receptor accessory protein. J Immunol. 2000;164:5277-5286.

27. Iwata T, Fujii T, Morii K, Saito M, Sugiyama J, Nishio H, et al. Cytokine profile in cervical mucosa of Japanese patients with cervical intraepithelial neoplasia. Int $J$ Clin Onco. 2015;20:126-133.

28. Weber A, Wasiliew P, Kracht M. Interleukin-1 (IL-1) pathway. Sci Signal. 2010;3:cm1.

29. Dunne A, O'Neill LAJ. The interleukin-1 receptor/Toll-like receptor superfamily: signal transduction during inflammation and host defense. Sci. STKE. 2003; (171): re3-re3.

30. Wan F, Lenardo MJ. The nuclear signaling of NF-kappaB: current knowledge, new insights, and future perspectives. Cell Res. 2010;20:24-33.

31. Naugler WE, Karin M. NF-kappaB and cancer-identifying targets and mechanisms. Curr Opin Genet Dev. 2008;18:19-26.

32. Gholamin S, Mitra SS, Feroze AH, Liu J, Kahn SA, Zhang M, et al. Disrupting the CD47-SIRP alpha anti-phagocytic axis by a humanized anti-CD47 antibody is an efficacious treatment for malignant pediatric brain tumors. Sci Transl Med 2017; 9:pii: eaaf2968.

33. Mehta AM, Mooij M, Brankovic I, Ouburg S, Morre SA, Jordanova ES. Cervical carcinogenesis and immune response gene polymorphisms: a review. J Immunol Res. 2017;2017:8913860.

34. Jabonowska-Fudziska D, Marszak A, Szylberg L, Tobacco smoking as a cofactor for the development of cervical cancer. Przegl Lek. 2014;72(3):103-105.

35. Li H, Cheng YM, Wu WW, Liu YG, Wei N, Feng XY, et al. SRSF10 regulates alternative splicing and is required for adipocyte differentiation. Mol Cell Biol. 2014;34:2198-2207.

36. Zhou XX, Li XB, Cheng YM, Wu WW, Xie ZQ, Xi QL, et al. BCLAF1 and its splicing regulator SRSF10 regulate the tumorigenic potential of colon cancer cells. Nat Commun. 2014; $5: 4581$.

37. Zhang W, Shen Q, Shen JX. Expression of neural salient serine-/ arginine-rich protein 1 (NSSR1) in colorectal cancer. J Zhejiang Univ Med Sci. 2011;40:540-544.

38. Peng ZY, Xiao PJ, Qi Y, Zhang W, Chen XH, Xu P. NSSR1 is regulated by testosterone in the mouse uterus and extensively expressed in endometrial carcinoma. Tumor Biol. 2011;32:359-366.

39. Senapati R, Senapati NN, Dwibedi B. Molecular mechanisms of HPV mediated neoplastic progression. Infect Agents Cancer. 2016;11:59.

40. Coussens LM, Werb Z. Inflammation and cancer. Nature. 2002;420:860-867.

41. Subramaniam S, Stansberg C, Cunningham C. The interleukin-1 receptor family. Dev Comp Immunol. 2004;28:415-428.

42. Gemma A, Takenaka K, Hosoya Y, Matuda K, Seike M, Kurimoto $\mathrm{F}$, et al. Altered expression of several genes in highly metastatic subpopulations of a human pulmonary adenocarcinoma cell line. Eur J Cancer. 2001;37:1554-1561.

43. Agerstam H, Karlsson C, Hansena N, Sanden C, Askmyra M, von Palffy S, et al. Antibodies targeting human IL1RAP (IL1R3) show therapeutic effects in xenograft models of acute myeloid leukemia. Proc Natl Acad Sci USA. 2015;112:10786-10791.

44. Karin M, Greten FR. NF kappa B: linking inflammation and immunity to cancer development and progression. Nat Rev Immunol. 2005;5:749-759.

45. Karin M. Nuclear factor-kappa B in cancer development and progression. Nature. 2006;441:431-436.

46. Da Costa RMG, MMSM Bastos, Medeiros R, Oliveira PA, The NF kappa B signaling pathway in papillomavirus-induced lesions: friend or foe?. Anticancer Res. 2016;36:2073-2083.

47. Sounni NE, Noel A. Targeting the tumor microenvironment for cancer therapy. Clin Chem. 2013;59:85-93.

48. Casey SC, Amedei A, Aquilano K, Azmi AS, Benencia F, Bhakta $\mathrm{D}$, et al. Cancer prevention and therapy through the modulation of the tumor microenvironment. Semin Cancer Biol. 2015;35: S199-S223.

49. Lo J, Lau EYT, Ching RHH, Cheng BYL, Ma MKF, Ng IOL, et al. Nuclear factor kappa B-mediated CD47 up-regulation promotes sorafenib resistance and its blockade synergizes the effect of sorafenib in hepatocellular carcinoma in mice. Hepatology. 2015;62:534-545.

50. Adamia S, Pilarski PM, Bar-Natan M, Stone RM, Griffin JD. Alternative splicing in chronic myeloid leukemia (CML): a novel therapeutic target? Curr Cancer Drug Target. 2013;13:735-748.

51. Jiang SH, Li J, Dong FY, Yang JY, Liu DJ, Yang XM, et al. Increased serotonin signaling contributes to the warburg effect in pancreatic tumor cells under metabolic stress and promotes growth of pancreatic tumors in mice. Gastroenterology. 2017;1:277-291.

52. Wen SY, Lin Y, Yu YQ, Cao SJ, Zhang R, Yang XM, et al. miR506 acts as a tumor suppressor by directly targeting the hedgehog pathway transcription factor Gli3 in human cervical cancer. Oncogene. 2015;34:717-725.

53. Liu R, Wei H, Gao P, Yu H, Wang K, Fu Z, et al. CD47 promotes ovarian cancer progression by inhibiting macrophage phagocytosis. Oncotarget. 2017;24:39021-3032.

54. Zeng D, Sun Q, Chen A, Fan J, Yang X, Xu L, et al. A fully human anti-CD47 blocking antibody with therapeutic potential for cancer. Oncotarget. 2016;7:83040-80350.

55. Dai M, Zhu XL, Liu F, Xu QY, Ge QL, Jiang SH, et al. Cholesterol synthetase dhcr24 induced by insulin aggravates cancer invasion and progesterone resistance in endometrial carcinoma. Sci Rep. 2017;7:41404. 